These are proofs of the final publication available at Springer: https://doi.org/10.1007/978-3-531-94259-9_13

\title{
Der geisteswissenschaftliche Bildungsdiskurs der preußischen Universitätsreform. Versuch einer wissenssoziologische Feld- und Diskursanalyse
}

Julian Hamann

\section{Einleitung}

Verglichen mit der gründlichen Erforschung der Naturwissenschaften blieben die Geisteswissenschaften als Untersuchungsgegenstand der Wissens- und Wissenschaftssoziologie stets merkwürdig vernachlässigt. Mit der Analyse des geisteswissenschaftlichen Bildungsdiskurses der preußischen Universitätsreform ermöglicht der Beitrag ein besseres Verständnis der Entwicklung der Geisteswissenschaften und stellt so auch einen Referenzpunkt für Analysen zum aktuellen Zustand der disziplinären Gruppe dar.

In einer wissenssoziologischen Feld- und Diskursanalyse werden sowohl der Diskurskontext in seiner materiellen und symbolischen Dimension als auch die konkrete diskursive Konstitution des Bildungsbegriffes erfasst und produktiv aufeinander bezogen. Nach einer einleitenden theoretischen Fundierung der Untersuchung werden unter Punkt zwei die Kategorien der Analyse des Feldes und des Diskurses in einem methodologischen Abschnitt vorgestellt. Das Feld als Kontext des Diskurses und der Bildungsdiskurs selbst werden dann im Hauptteil unter den Punkten drei und vier behandelt. Punkt fünf schließt mit einer Zusammenführung der beiden Analyseteile.

\section{Die Selbstreflektion im geisteswissenschaftlichen Feld}

Die so genannte Krise der Geisteswissenschaften ist keineswegs ein neues Phänomen. Zumindest in den Geisteswissenschaften selbst existiert seit jeher eine eigene Literaturgattung, die sich damit befasst, was die Geisteswissenschaften eigentlich sind, wie man über die Disziplinengruppe sprechen darf und in welchem Zustand 
sie sich befindet. ${ }^{1}$ Eine zentrale Referenz dieser ausführlichen Selbstbeobachtung ist das legitime Konzept geisteswissenschaftlicher Bildung. Wird dieser vorerst mit dem ungefähren Begriff Bildungsdebatte zu bezeichnende Diskussionszusammenhang aus einer diachronen Perspektive betrachtet, dann zeigt sich, dass sich die disziplinäre Identität der Geisteswissenschaften als Fixpunkt ihrer Selbstreflektion bereits vor rund 200 Jahren ausgebildet hat. Im Umfeld der preußischen Universitätsreform sind es vornehmlich Philosophen und Philologen, die die Debatten um einen legitimen Entwurf der Universität und die damit verbundene Idee wissenschaftlicher Bildung dominieren. In diesen Auseinandersetzungen bildet sich ein neuhumanistisch-idealistisches Selbstverständnis aus, von dem die geisteswissenschaftliche Selbstwahrnehmung noch heute beeinflusst ist. ${ }^{2}$ Ein Verständnis der weiteren Entwicklung der Disziplinengruppe, deren Selbstbild seither durch Abgrenzungen von den Naturwissenschaften sowie von einer regelmäßig aufflammenden Krisenstimmung gezeichnet ist, setzt daher die Bildungsdebatten zu Beginn des 19. Jahrhunderts als Referenzpunkt voraus. Die Bildungsdebatte der preußischen Universitätsreform stellt demnach einen sinnvollen Startpunkt für etwaige anschließende Überlegungen dar (vgl. Hamann 2009, 2011).

Eine soziologische Analyse der Auseinandersetzung um den legitimen Bildungsbegriff muss zum einen den institutionellen Rahmen der geisteswissenschaftlichen Selbstbeobachtung erfassen können. Dies gilt umso mehr, wenn der Untersuchungsgegenstand keine quer zur institutionellen Ordnung der Universität liegende Intellektuellenschicht ist, sondern in festen akademischen Strukturen verankert ist (vgl. Kauppi 1996; Angermüller 2007). Zum anderen scheint es ge-

1 Fragen wie Wozu Geisteswissenschaften? (Keisinger/Seischab 2003), Wie sozial ist der Geist der Geisteswissenschaften? (Koselleck 1991) oder Wes Geistes Wissenschaften? (Schilling/Wiedemann 1989) sowie Reflektionen über Probleme und Perspektiven der Geisteswissenschaften (Malinowski 2006), Zur Situation der Geisteswissenschaften (Wehler 1998), zum Glanz und Elend der Geisteswissenschaften (Mittelstraß 1989) und Über die Unvermeidlichkeit der Geisteswissenschaften (Marquard 1986a) oder die Aufgabe der Geisteswissenschaften in der modernen Gesellschaft (Ritter 1974a) können dabei genauso auf eine reiche Tradition von Abhandlungen Über den Vortrag der Philosophie an den Universitäten (Hegel 1990[1816]) und Über die Idee der Universitäten (Steffens 1910[1809]), auf Gedanken über Universitäten im deutschen Sinn (Schleiermacher 1956[1808]) und Schriften über die Bestimmung des Gelehrten (Fichte 1845[1794]) sowie über den Streit der Fakultäten (Kant 2005[1798]) zurückgreifen, wie eine Theorie der Unbildung (Liessmann 2006) auf einer Theorie der Halbbildung (Adorno 2006[1959]) und wohl letztlich auf einer Theorie der Bildung des Menschen (Humboldt 1960[1793]) aufbaut.

2 Der Literaturwissenschaftler Wolfgang Frühwald (1991: 73) bemerkt: „Die Fächer und Disziplinen der Geisteswissenschaften sind heute [...] noch immer stärker und nachhaltiger auf die aus dem Geist des Idealismus und des Neuhumanismus geborene Universität des 19. Jahrhunderts bezogen, als dies im Alltag des Hochschullebens bewußt wird." Dies gilt natürlich, obwohl der Begriff „Geisteswissenschaften“ sich erst in der zweiten Hälfte des 19. Jahrhunderts in systematisierter Form durchsetzt (vgl. Diemer 1974: 212; Geldsetzer 1974: 143). 
rade für Untersuchungen intellektueller Zusammenhänge wichtig, für die symbolische Dimension sozialer Praxis sensibel zu sein. So besteht die Möglichkeit, der vermeintlichen Interessenlosigkeit wissenschaftlicher Praxis analytisch Rechnung zu tragen. Schließlich ist es von Bedeutung, die Beziehung zwischen dem institutionellen Rahmen und den darin produzierten Kulturgütern zu erfassen und damit sowohl eine rein textimmanente Lektüre als auch die direkte Rückführung kultureller Erzeugnisse auf externe Faktoren zu vermeiden.

Mit seinen Arbeiten zu intellektuellen, universitären und wissenschaftlichen Feldern hat Bourdieu $(1969,1992,1998$ b, 2004) ein theoretisches Konzept entwickelt, das diesen Ansprüchen an eine Analyse der Bildungsdebatte gerecht werden kann.

Die Besonderheit des geisteswissenschaftlichen Feldes liegt nun erstens darin, dass die weltlichen und wissenschaftlichen Hierarchisierungsprinzipien eine ausgeprägt chiastische Struktur aufweisen. Eine Folge dieser Eigenheit ist, dass das geisteswissenschaftliche Feld über einen besonders autonomen Pol verfügt. Mehr noch als in anderen wissenschaftlichen Feldern gilt hier die Illusio interessenlosen Interesses, einer bedingungslosen und uneigennützigen Hingabe an reine wissenschaftliche Erkenntnis. Die stark ausgeprägte Unvereinbarkeit wissenschaftlicher und weltlicher Macht wird dadurch ergänzt, dass zweitens beide Machtformen in einem besonders ausgeglichenen Verhältnis stehen. Neben der spezifisch wissenschaftlichen Logik einer vermeintlich reinen wissenschaftlichen Wahrheit steht daher die starke Präsenz einer weltlichen Macht am entgegengesetzten Pol. Dafür sorgen in den Geisteswissenschaften die sozialen Funktionen der Wahrung und Kanonisierung von Bildungsinhalten sowie das weitgehende Monopol auf die Ausbildung von Lehrern. Zwischen diesen beiden Polen findet die nicht-selbstreflexive Praxis des geisteswissenschaftlichen Feldes statt. Ergänzt wird das Nebeneinander zweier Machtformen drittens durch intellektuelle oder literarische Prominenz, die als Variation der wissenschaftlichen Macht eine dem geisteswissenschaftlichen Feld eigentümliche Art von Kapital darstellt (Bourdieu 1992: 68, 133, 1998b: 27, 36-37, 2004: 57). Das so umrissene Konzept des geisteswissenschaftlichen Feldes erlaubt es, die Produktion und Rezeption von Beiträgen der Bildungsdebatte in eine Kontextstruktur einzubetten, die aus einer materiellen Dimension der Verteilung und einer symbolischen Dimension der Bewertung von Ressourcen besteht. Ausdeutungen des Bildungsbegriffes erscheinen in dieser Struktur als symbolische Güter und die Reflektionen der am Diskurs beteiligten Geisteswissenschaftler präsentieren sich als symbolische Auseinandersetzungen, bei denen um die legitime Definition wissenschaftlicher Bildung gerungen wird. 
Das skizzierte Gefüge feldtheoretischer Begriffe und Konzepte sollte jedoch nicht als fester Fahrplan für die Durchführung einer unvermittelt an Bourdieu anschließenden Untersuchung verstanden werden. Vielmehr handelt es sich um einen Baukasten heuristischer Werkzeuge, die sich erkenntnisleitend und strukturierend zur Analyse der geisteswissenschaftlichen Bildungsdebatte einsetzen lassen. Durch die Anpassung dieser Werkzeuge an die Erfordernisse und Einschränkungen einer historischen Fragestellung kann die feldanalytische Orientierung produktiv in die Forschungspraxis umgesetzt werden.

\section{Die Kategorien der Analyse des Feldes und des Diskurses}

Die feldanalytische Erfassung des Produktions- und Rezeptionskontextes der Bildungsdebatte konzentriert sich auf eine Auswahl relevanter Kontextfaktoren. Anhand der konkreten Ausprägung dieser Faktoren können dann Annahmen über den jeweiligen Zustand des Debattenkontextes gemacht werden. Die Analyse fokussiert erstens die materielle Verteilungsstruktur. Ihr nähert sich die Untersuchung durch zwei institutionelle Merkmale des Feldes, die als Faktoren Aussagen über die Allokation diskursrelevanter Ressourcen ermöglichen. Der Faktor Population umfasst Zahl und soziale Herkunft der Studenten, Umfang und Rekrutierungsmodus der Professorenschaft sowie das daraus resultierende Betreuungsverhältnis. Beabsichtigt ist die sozialstrukturelle Annäherung an die für geisteswissenschaftliche Bildung wichtigsten Akteursgruppen. Der institutionelle Faktor umfasst Zahl und Wachstum von Fächern und Lehrstühlen sowie weitere organisatorische Merkmale. Hier ist das Ziel, den im engeren Sinne institutionellen Rahmen geisteswissenschaftlicher Bildung zu erfassen.

Kombiniert werden diese Merkmale zweitens mit Faktoren einer wissenssoziologisch gewendeten Feldanalyse (vgl. für diese Akzentsetzung Angermüller 2007: 45; siehe aber auch die Überlegungen zum „Raum des Möglichen“ in Bourdieu 1998a: 55-56). Die symbolische Dimension des Feldes erscheint dann als Referenzraum der Bildungsdebatte. Damit sind die etablierten (jedoch keineswegs zwingend unumstrittenen) Wissensbestände gemeint, auf die bei der Produktion und Rezeption von Debattenbeiträgen zurückgegriffen werden kann. Diesem symbolischen Bewertungshorizont nähert sich die Untersuchung durch zwei Faktoren. Der Faktor Wissenschaftsverständnis umfasst als wissenschaftlich legitim verstandene Methoden und Untersuchungsgegenstände, Qualitätsmerkmale und Standards. Indem dieses Wissen erfasst wird, erhält neben den Reflektionen über Bildung ein weiterer elementarer Bestandteil der Selbstbeobachtung in den Geisteswissenschaften Gewicht in der Analyse. Der Faktor Akteurspositionen umfasst 
Zuschreibungen über, den Studenten “ und ,den Professor' als idealtypische Positionen in der Struktur des Feldes. Hier soll das verfügbare Wissen über die beiden wichtigsten Akteursgruppen geisteswissenschaftlicher Bildung katalogisiert werden. ${ }^{3}$

Mit Hilfe dieser Faktoren lässt sich die materielle und symbolische Struktur des Debattenkontextes systematisch untersuchen. Es ist entscheidend, dass die vier ausgewählten Strukturmerkmale von hoher Relevanz für die Auseinandersetzungen um geisteswissenschaftliche Bildung sind. So wird gewährleistet, dass sich die von den Faktoren angezeigten strukturellen Veränderungen in der disziplinären Selbstreflektion niederschlagen. Die durch diese Faktoren geleitete Analyse kann, unter Rückgriff auf ein interpretativ-analytisches Instrumentarium und in den Grenzen, die durch die Quellen- und Datenlage gesetzt sind, das geisteswissenschaftliche Feld als Produktions- und Rezeptionskontext der Bildungsdebatte skizzieren.

Wird Bourdieus (1988: 10, 1998b: 17-18) Anspruch, sowohl einer Illusion der Autonomie wissenschaftlicher Texte als auch einer direkten Rückführung der Texte auf ihren sozialen Kontext eine Absage zu erteilen, tatsächlich ernst genommen, dann kann die Analyse nicht an diesem Punkt stehen bleiben. Trotz dieses Anspruchs hat Bourdieu selbst sich stets eher für den sozialen Kontext der Produktion von Kulturgütern interessiert. Seine Annahme, „daß die allerspezifischsten Merkmale des Diskurses [...] auf die sozialen Bedingungen seiner Produktion zurückzuführen sind“, führt ihn dann auch zu folgendem Schluss: ,[W]as eigentlich zu analysieren wäre, sind die sozialen Bedingungen der Konstituierung des Feldes, in dem dieser Diskurs produziert wird, denn dort liegt das eigentliche Prinzip dessen, was hier gesagt oder nicht gesagt werden konnte.“ (Bourdieu 1993a: 131, 134) Da für ihn jede diskursive Äußerung lediglich eine „Manifestation“ der objektiven Strukturen ist, hat Bourdieu (2006: 290) nie systematisch Instrumente zur Analyse des konkreten Inhalts von Kulturgütern entwickelt (zu einem ähnlichen Urteil kommt Diaz-Bone 2002: 69-70). Auch wenn mit Die politische Ontologie Martin Heideggers (Bourdieu 1988, vgl. a. 2001: 19-79) eine beeindruckende Fallstudie dieser Perspektive existiert, erfordert die inhaltliche Analyse der Auseinandersetzungen um geisteswissenschaftliche Bildung daher eine Erweiterung des feldanalytischen Rahmens. Dokumentiert sich die historische Praxis wie im vorliegenden Fall in erster Linie in schriftenbasierten Kommunikationsformen, ist die Erfassung der textuellen Inhaltsebene als eigenständigem empirischen Datum umso drängender.

3 Die Analyse feldintern getätigter Annahmen über idealtypische Akteurspositionen eignet sich besser als das theoretisch (und empirisch) voraussetzungsvollere Habituskonzept, um die für die Bildungsdebatte relevanten Wissensvorräte zu katalogisieren. Der Faktor Akteurspositionen erfasst daher nicht den gesamtgesellschaftlich wirksamen und stärker sozialstrukturell verankerten Habitus, sondern lenkt den Blick auf die im Rahmen der Fragestellung relevanteren, weil von Mitstreitern im Feld zugeschriebenen Manifestationen eines Habitus. 
Der Zugang zu dieser inhaltlichen Ebene der Selbstreflektion über Bildung ist vor allem an der Wissenssoziologischen Diskursanalyse orientiert (Keller 2008). Aufgrund gemeinsamer methodologischer und strukturationstheoretischer Grundannahmen kann das auch an Foucault anschließende Forschungsprogramm produktiv mit Bourdieus Feldanalyse verknüpft werden (vgl. a. Schmidt-Wellenburg 2011). Die Bildungsdebatte lässt sich so als Bildungsdiskurs verstehen, als Sinnund Strukturierungszusammenhang, den es reflektiert und systematisch zu rekonstruieren und zu interpretieren gilt (vgl. Dreyfus/Rabinow 1987; Keller 2005). Symbolische Auseinandersetzungen werden dann zu (Be-)Deutungsarbeit, deren Ziel die interpretative Hegemonie im Diskurs ist. In den Auseinandersetzungen etablierte Definitionen geisteswissenschaftlicher Bildung kulminieren zu überindividuellen Wissensordnungen, die als strukturierte und strukturierende Strukturen die Praxis der am Diskurs beteiligten Akteure generieren und gleichzeitig im Rahmen dieser Praxis generiert werden (vgl. Keller 2005: 64). Als strukturierte Struktur sind legitime Definitionen von Bildung das Ergebnis einer konflikthaften diskursiven Konstruktion, als strukturierende Struktur wirken sie auf den Diskurs zurück, weil sie relevantes Wissen konstituieren und damit einen Bezugspunkt für neue diskursive Auseinandersetzungen darstellen.

Die Wissenssoziologische Diskursanalyse hält verschiedene heuristische Instrumente zur Untersuchung der inhaltlichen Strukturierungsebene bereit. Hervorgehoben werden soll hier die Phänomenstruktur (Keller 2008: 248-251). Grundlegender Gedanke hinter diesem Konzept ist, dass diskursive Gegenstände aus verschiedenen Elementen bestehen, die im Prozess ihrer Konstruktion in unterschiedlichen Kombinationen aktiviert und zu einer spezifischen Gestalt verbunden werden können. Die Phänomenstruktur ist der jeweils diskursiv vorgegebene Möglichkeitsraum, innerhalb dessen individuelle Ausdeutungen stattfinden können. Daran anschließend wird Bildung hier als diskursiver Gegenstand verstanden, der sich auf fünf Dimensionen entfaltet, auf denen wiederum verschiedene Elemente angerufen werden können. Die ontologische Dimension versammelt unterschiedliche Deutungen zum Wesen, zum Charakter oder zur Definition von Bildung. Die funktionale Dimension erfasst verschiedene Zuschreibungen zum Zweck oder Nutzen geisteswissenschaftlicher Bildung. Auf der Objektdimension finden sich Interpretationen zu Gegenständen oder Objekten, an denen sich Bildung vollzieht. Die praktische Dimension enthält Deutungen über konkrete Formen und praktische Bedingungen geisteswissenschaftlicher Bildung. Schließlich erfasst die Subjektdimension Zuschreibungen von Verantwortungen oder Eigenschaften unterschiedlicher Subjektpositionen im Bildungsdiskurs. Die induktiv hergeleitete Phänomenstruktur zielt auf die systematische Rekonstruktion und Interpretation 
diskursiv erzeugter Wissensordnungen ab. Ziel dieser diskursanalytischen Ergänzung des feldanalytischen Rahmens ist es, konkreten Inhalten eine größere Bedeutung einzuräumen und sie als eigenständige Strukturierungsebene zu begreifen.

\section{Der Bildungsdiskurs und sein Kontext}

Im Folgenden soll eine empirische Untersuchung die forschungspraktische Umsetzung des skizzierten Analyserahmens illustrieren. Zunächst wird unter Punkt 3.1 das geisteswissenschaftliche Feld als Kontext des Bildungsdiskurses analysiert. Die jeweilige Beschaffenheit der materiellen und symbolischen Struktur des Feldes ermöglicht dann Annahmen über den Grad der quantitativen und qualitativen Geschlossenheit der Verteilungs- und Bewertungsstruktur sowie über den Autonomiegrad des Feldes. Unter Punkt 3.2 folgt die Analyse des in dieser Struktur situierten Bildungsdiskurses. Mit dem Begriff der Phänomenstruktur kann die Gestalt des Bildungsbegriffes auf fünf Dimensionen systematisch rekonstruiert werden. Dies geschieht anhand von Beiträgen, die zu den wichtigsten Äußerungen im geisteswissenschaftlichen Bildungsdiskurs zum Zeitpunkt der preußischen Universitätsreform gezählt werden können.

Die Reform findet vor dem Hintergrund einer Krise der Universitäten am Ende des 18. Jahrhunderts statt, einem Wendepunkt, nach dem das universitäre Feld weitgehend neu bestellt werden muss. Zwar gehören die Neugründungen in Halle (1694) und Göttingen (1737) schon bald zu den modernsten Universitäten Deutschlands, jedoch sind sie keinesfalls repräsentativ für die Mehrzahl der deutschen Universitäten, die eben nicht im Geiste der Aufklärung neu gegründet werden. Diese nicht selten existenzgefährdend kleinen und provinziellen Institutionen vereinen traditionelle Elemente, die bis in das Mittelalter zurückreichen, mit dem neuen Aufklärungsdenken. So finden sich an den Universitäten immobile, von der Forschung entfremdete und sich dem Standesdünkel hingebende Professoren, schlecht auf das Studium vorbereitete, zügellos lebende Studenten und verkrustete Curricula sowie nepotistische und vom Staat oktroyierte Berufungsverfahren kombiniert mit dem Nützlichkeitsdenken der Aufklärung und ihrer ausgeprägten Orientierung an alltäglichen Problemen. Unter diesen beiden Einflüssen verlieren Universitäten den Anschluss an ihre Zeit und verkümmern - eine Entwicklung, von der die philosophischen Fakultäten (oder Artistenfakultäten) besonders getroffen sind. Die Säkularisierung, der Untergang des Alten Reiches und die napoleonischen Kriege dezimieren die auf diese Weise geschwächte Institution so stark, dass 1818 nur mehr die Hälfte der 1792 noch vorhandenen 42 deutschen Universitäten existiert (vgl. 
McClelland 1980: 27-98; Fuhrmann 1997: 31-34; für einen positiveren Blick auf die Aufklärungsuniversität siehe aber auch Füssel 2010: 65-70).

\subsection{Der Produktions- und Rezeptionskontext des Diskurses}

\subsubsection{Der Faktor Population}

Das zurückgehende Ansehen der krisengeschwächten Universitäten in der zweiten Hälfte des 18. Jahrhunderts spiegelt sich im deutlich sinkenden Anteil der Studenten an der Gesamtbevölkerung wider. Zwischen 1815 und 1830 verdreifacht sich die Studentenzahl dann jedoch auf etwa 16.000 (Eulenburg 1904: 187, 269; Jarausch 1981: 16-23). An dieser Entwicklung sind geisteswissenschaftliche Fächer maßgeblich beteiligt: In Preußen steigt der Anteil der Studenten in den Sprachund Kulturwissenschaften an der Gesamtzahl der Studenten im Zeitraumvon 1820 bis 1840 von $15 \%$ auf $26 \%$ (Titze 1987: 27-30, 86-101, den Hauptanteil hält hier noch die Theologie).

Dieser Anstieg ist zumindest in diesem Ausmaß politisch nicht gewollt. Die Furcht vor der Entwertung akademischer Bildung durch ein akademisches Proletariat sorgt für eine Verschärfung der sozialen Auslese durch striktere Zugangsvoraussetzungen (Nipperdey 1987: 478; Titze 1999: 355, 364-365). Gegen den politischen Willen hält dieser Anstieg der Studentenzahlen etwa bis zur Mitte des 19. Jahrhunderts an. Zumindest die Anfangsphase dieser Expansion kann mit der Kompensation des Studentenmangels in dem Vierteljahrhundert von der Französischen Revolution bis zum Wiener Kongress erklärt werden - die angesichts der Mangelsituation hohen Chancen auf Beschäftigung und der hohe Distinktionswert universitärer Bildung zeitigen Sogeffekte, die für eine relative Normalisierung der Studentenzahlen sorgen (Titze 1985). Für die Fortsetzung des Wachstums spielt dann aber das im Rahmen der preußischen Reformen erneuerte Prestige der universitären Bildung eine entscheidende Rolle. In der Folge dieser Aufwertung wird Bildung zu einem effektiven Distinktionsmittel und stellt im Wettbewerb um die Privilegien des Beamtentums den einzigen Ersatz für eine hohe Geburt dar (Jarausch 1981: 19-20).

Obwohl die Bildungsidee der Reformuniversität zumindest theoretisch unabhängig von der sozialen Herkunft ist, hat sie einen implizit exklusiven Charakter, weil die Verachtung des Brotstudiums eine gute schulische Bildung und die materielle Möglichkeit zur Muße voraussetzt. Es überrascht daher nicht, wenn in der ersten Hälfte des 19. Jahrhunderts über $80 \%$ der Studenten aus bürgerlichen Elternhäusern stammen und über $50 \%$ Väter mit akademischer Bildung haben. Innerhalb der Gruppe der Studenten aus dem Bildungsbürgertum sind fast viermal 
mehr Beamtensöhne anzutreffen als Nachkommen freier Akademiker, was auf die zentrale Rolle des Staates als Arbeitgeber der Absolventen hinweist. Ein dynamischer Vergleich der sozialen Herkunft in den ersten beiden Dritteln des 19. Jahrhunderts zeigt, dass der Anteil der Studenten aus dem Bildungsbürgertum zwar zunächst sinkt, dann aber auf weiterhin hohem Niveau knapp unter 50\% stagniert. Den deutlichsten Zuwachs erfährt in dieser Zeit das Besitzbürgertum, dessen Anteil von etwa $7 \%$ auf fast $30 \%$ ansteigt (Jarausch 1981: 32-44).

Auch die Zahl des Lehrpersonals befindet sich zu Beginn des 19. Jahrhunderts auf einem Tiefpunkt. Relativ zur Studentenzahl lassen sich im letzten Drittel des 18. Jahrhunderts zwar mehr Lehrkräfte zählen als in den ersten Jahrzehnten des 19. Jahrhunderts. Die ab den 1820er Jahren einsetzende Expansion der Studentenzahlen findet aber keine Entsprechung bei den Professuren. Insgesamt steigt die Zahl der Professoren sehr viel langsamer als die Zahl der Studenten. Diese Entwicklung muss im Zusammenhang mit dem Anstieg der außerordentlichen Lehrkräfte gesehen werden, der jedoch ebenfalls nur einen Teil der Studentenzahlen auffangen kann, so dass sich die Betreuungsrelationen insgesamt verschlechtern (Titze 1995: 62, 66). Wie bei den Studentenzahlen nehmen die Geisteswissenschaften aber auch beim Lehrpersonal eine Sonderrolle ein. Eulenburg (1904: 248) bemerkt für den Beginn des 19. Jahrhunderts, dass ,der Löwenanteil der neuen Professuren [...] auf die philosophische Fakultät"“ entfällt. Dass den geisteswissenschaftlichen Ordinarien eine besondere Bedeutung zukommt, zeigt neben Eulenburg auch Baumgarten (1997: 50), die eine bemerkenswerte Förderung der philologisch-historischen Fächer zu Beginn des 19. Jahrhunderts verzeichnet.

Die Besetzung dieser Lehrstühle erfolgt aus eng begrenzten sozialen Kreisen. Laut Baumgarten (1997: 93) ist die soziale Verflechtung in den Geisteswissenschaften zu Beginn des 19. Jahrhunderts überaus dicht. Fast die Hälfte aller Geisteswissenschaftler ist mit einem weiteren Dozenten verwandt oder verschwägert. Nahezu 10\% haben einen Professor zum Vater, ebenfalls $10 \%$ sind mit einer Professorentochter verheiratet. Jeder siebte Professor hat einen oder mehrere Söhne, die ebenfalls das akademische Lehramt anstreben. Eine besondere Rolle spielen Universitätsfamilien, in denen ein akademisches Lehramt über mehr als zwei Generationen ausgeübt wird. ${ }^{4}$ Diese familiären Kristallisationspunkte spielen, so

4 Baumgarten (1997: 93-102) verweist für die sechs von ihr untersuchten Universitäten auf zehn Universitätsfamilien im engeren Sinne, die sich vom 17. bis zum 19. Jahrhundert ausbilden. Die in Wittenberg und Kiel ansässige Familie Nitzsch bringt, vom 17. Jahrhundert ausgehend, über fünf Generationen hinweg Rechtswissenschaftler, Theologen und Altphilologen hervor, darunter den bedeutenden Theologen Karl Ludwig Nitzsch (1751-1831). Die Abkömmlinge der Heidelberger Universitätsfamilie Wundt besetzen ab Mitte des 18. Jahrhunderts über vier Generationen hinweg fünf Professuren in der theologischen, der philosophischen und der medizinischen Fakultät. Der bekannte Physiologe und Psychologe Wilhelm Wundt heiratet 1872 Luise Mau, die Tochter des 
Baumgarten (1997: 130, 2001: 112), in den Naturwissenschaften eine wesentlich geringere Rolle als in den Geisteswissenschaften. Ein Blick auf die soziale Herkunft der Geisteswissenschaftler zeigt, dass in der ersten Hälfte des 19. Jahrhunderts über $50 \%$ des Lehrpersonals aus dem beamteten Bildungsbürgertum stammen. Ähnlich wie bei den Studenten wird dieser Anteil im Laufe des Jahrhunderts leicht sinken, sich aber auf weiterhin hohem Niveau einpendeln. Die zweitgrößte Herkunftsschicht ist die untere Mittelschicht mit etwa 20\%. Freiberufliches und besitzendes Bürgertum stellen jeweils etwa $8 \%$ der geisteswissenschaftlichen Professoren (Baumgarten 1997: 287-290).

\subsubsection{Der institutionelle Faktor}

Wenn von einem in der preußischen Universitätsreform geschaffenen neuen Typus der Universität die Rede ist, dann betrifft dies nicht das korporationsrechtliche Modell der Senats- und Fakultätenverfassung. Vielmehr sollen die Reformen ein „altehrwürdiges, aber verstaubt erscheinendes Gehäuse mit neuem Geist“ (Bruch 1999: 392) erfüllen und es so von innen heraus erneuern. Die Gründung in Berlin findet schnell Nachahmer. ${ }^{5}$ So werden zunächst Breslau und dann 1818 die Neu- und Wiedergründung in Bonn nach preußischem Vorbild organisiert. Auch Würzburg, Heidelberg und die Universität Landshut, die 1825/26 nach München kommt, bilden sich allmählich um. Diese Universitäten erlangen ein beachtliches Prestige, nach 1815 studieren etwa 40\% aller Studenten an den vier großen Universitäten in Berlin, Breslau, Bonn und Landshut/München (Nipperdey 1987: 471).

Wenn auch die grundsätzliche Fakultätsstruktur beibehalten wird, so erfährt ihre interne Hierarchie einen fundamentalen Wandel. Die noch im 18. Jahrhundert verfallene philosophische Fakultät profitiert materiell und symbolisch von der Professionalisierung der Lehrerbildung, durch die ihr ein eigener Berufsstand zuwächst (vgl. Tenorth 1987). Ihr allgemeinbildender Charakter, der sie zuvor zu einer Vorschule der übrigen drei Fakultäten degradierte, wird im Lichte des sich wandelnden Bildungsideals zu einem entscheidenden Vorteil im Streit der Fakul-

Theologieprofessors Heinrich August Mau und Schwester des Archäologen August Mau. Die in Marburg beheimatete Familie Justi bringt über fünf Generationen hinweg fünf Professoren hervor, die sich in der Theologie, in der Sprachwissenschaft und, wie Carl Justi (1832-1912), als Kunsthistoriker profilieren. Als letztes Beispiel sei die Universitätsfamilie Bruns genannt, von der ab Mitte des 18. Jahrhunderts sechs Nachkommen über fünf Generationen hinweg eine akademische Laufbahn verfolgen.

5 Auch wenn sich die theoretische Gründungsidee nirgends in Reinform durchsetzt, dürfte an der Bedeutung der in Berlin vorgenommenen Universitätsgründung kein Zweifel bestehen (vgl. Schelsky 1971: 21; Ringer 1987: 33; siehe aber auch Bruch 1999: 392; Paletschek 2001: 2-7, 159-160). Dies gilt insbesondere für den vorliegenden Zusammenhang, weil sich erst mit der Gründung in Berlin die philosophische Fakultät als Leitfakultät durchsetzt. 
täten (Kant 2005[1798]). Es ist kein Zufall, dass viele häretische Konzepte, die gegen die Dominanz der anderen Fakultäten gerichtet sind, zuerst an der noch untergeordneten philosophischen Fakultät etabliert werden.

In den ersten Dekaden des 19. Jahrhunderts zeichnet sich dieser Wandel nicht nur am Anteil der philosophischen Fakultät am Zuwachs der universitären Population ab (vgl. a. Rüegg 2004a: 375-377). Die Universitätsreform geht einher mit der großzügigen Förderung philologisch-historischer Universitätsfächer. Besonders in Philosophie und Altphilologie kommt es zu zahlreichen Lehrstuhlgründungen. Auch Geschichtswissenschaft und Orientalistik erfahren als weitere geisteswissenschaftliche Kernfächer einen bemerkenswerten Ausbau. Diese Expansion ist zunächst auf den etablierten Kern der Disziplinen beschränkt, während eine Ausdifferenzierung in Fachschwerpunkte etwa ab der Jahrhundertmitte stattfindet. Erst dann werden auch Lehrstühle für neue Fächer wie Slawistik, Ägyptologie oder Musikwissenschaften gegründet (vgl. die Gründungsjahre geisteswissenschaftlicher Lehrstühle in Baumgarten 1997: 277-281; s. a. die äußerst detaillierte Studie zur Geschichtswissenschaft von Weber 1984: 53). Im Vergleich mit dem Ausbau der Naturwissenschaften zeigt sich, dass sich der wissenschaftsgeschichtliche Vorsprung der Geisteswissenschaften an fast allen Universitäten auch in einem bevorzugten institutionellen Ausbau niederschlägt. Bis etwa zur Mitte der 1830er Jahre liegt das Hauptgewicht der Lehrstuhlgründungen daher auf philologisch-historischen Fächern (Baumgarten 1997: 90; s. a. Rüegg 2004a: 348-361).

Welche Annahmen über die Struktur des geisteswissenschaftlichen Feldes zur Zeit der preußischen Universitätsreform ergeben sich angesichts dieser Ausführungen? Die materielle Struktur kann mit Blick auf die Population als quantitativ leicht geöffnet und qualitativ weitgehend geschlossen beschrieben werden. Quantitativ ist im unmittelbaren Nachklang der Universitätsreform ein leichter Anstieg der Studentenzahlen zu verzeichnen, während die Zahl der Professoren nur schwach, im Vergleich zu den übrigen Fakultäten aber dennoch nicht unwesentlich steigt. Im Rahmen der qualitativen Schließung kann sich das Bildungsbürgertum trotz eines leichten Rückgangs als wichtigste Rekrutierungsschicht von Professoren und Studenten halten. Mit Blick auf die institutionelle Ebene ist ebenfalls eine quantitative Öffnung zu erkennen. Die Lehrstuhlgründungen beschränken sich aber auf die geisteswissenschaftlichen Kernfächer. Ihre Ausdifferenzierung in weitere Fachbereiche sowie der Ausbau neuer Fächer erfolgen erst deutlich nach der Universitätsreform, so dass das Feld auch hier qualitativ geschlossen bleibt. 


\subsubsection{Der Faktor Wissenschaftsverständnis}

Die Reformära markiert den Übergang von einem an kanonisierten und statischen Wissensbeständen orientierten Wissenschaftsverständnis der Aufklärung zu einer Forschungsorientierung, die auf ständige Erweiterung der Wissenshorizonte drängt. Zwar knüpft das Wissenschaftsverständnis zunächst noch an die Aufklärung an, wenn neue Erkenntnisse in einen großen, allgemein akzeptierten Zusammenhang eingefügt und Entdeckungen auf bereits vorhandenes Wissen bezogen werden sollen. Wie auf institutioneller Ebene zu erkennen ist, differenzieren sich aber gerade die Geisteswissenschaften schon bald zu sehr aus, um diesem universalistischen Einheitsdenken weiterhin gerecht werden zu können. Spezialwissen kann nun nicht mehr auf eine alle Wissenschaften überspannende Einheit bezogen werden, so dass der statische Wissensbegriff als Referenzpunkt der Wissenschaften an Glaubwürdigkeit verliert.

Weil auf einen Kanon aufbauende wissenschaftliche Autorität vor dem Hintergrund sich schnell wandelnder Wissenshorizonte keine Orientierung mehr zu bieten scheint, muss Wissen über die Welt nun von jedem einzelnen Forscher selbst hervorgebracht werden (vgl. Hardtwig 1990: 18). Wilhelm von Humboldts ${ }^{6}$ (1956[1810]: 379) Grundsatz, „Wissenschaft als etwas noch nicht ganz Gefundenes und nie ganz Aufzufindendes zu betrachten, und unablässig sie als solche zu suchen" erfordert es, vorhandenes Wissen einer fortdauernden Prüfung zu unterziehen. Die so fokussierte Unbeständigkeit von Wissen führt dazu, dass es zu einer tendenziellen Abwendung von Resultaten und feststehendem Wissen und zu einer verstärkten Methodenorientierung kommt (vgl. McClelland 1983: 316). Diese Konzentration auf die reine wissenschaftliche Erkenntnis, die doch nicht dauerhaft sein kann, macht aus der Universität als Lehranstalt eine Forschungsuniversität.

Das dezidiert geisteswissenschaftliche Wissenschaftsverständnis der unmittelbaren Reformära äußert sich im Führungsanspruch der Philosophie innerhalb der Universität. Sie bildet den Nukleus einer zunächst noch universalistischen Wissenschaftsidee, aus dem sich alle anderen wissenschaftlichen Tätigkeiten ableiten (z. B. Fichte 1845[1794]: 328, 1910[1807]: 26-27, 35-37; Schleiermacher 1956[1808]:

$6 \quad$ Wilhelm von Humboldt (1767-1835) genießt als Sprössling einer vermögenden Familie eine äußerst umfangreiche Ausbildung und wird unter anderem von Johann Jakob Engel unterrichtet. Nach nur vier Semestern absolviert Humboldt das Studium der Rechtswissenschaften in Frankfurt/O. und Göttingen. Danach widmet er sich den Studien Kants und bleibt zeitlebens von ihm beeinflusst. Nach kurzem Staatsdienst ermöglicht ihm die finanzielle Sicherheit Reisen und einige (sprach-) philosophische Publikationen, bevor 1809 sein bedeutsames staatsmännisches Wirken beginnt. Im gleichen Jahr wird er Mitglied der Preußischen Akademie der Wissenschaften. Nach einem Jahr auf Gesuch entlassen ist Humboldt als Diplomat in verschiedenen europäischen Metropolen tätig, bevor er sich ab 1819 zurückzieht und sich ausschließlich seinen Studien widmet (Schmitz 1989). 
240, 260-262; Steffens 1910[1809]: 261; Hegel 1990[1816]: 289). ${ }^{7}$ Das Fach verkörpert die Trennung der Wissenschaft von ihrer Anwendung, ein Axiom der idealistisch begründeten Wissenschaftsauffassung, das Humboldt (2008[1814]: 920) wie folgt auf den Punkt bringt: ,Wo der Gedanke um des Gedankens willen entzückt, da führt echt wissenschaftlicher Sinn das Denken bis nahe zu seinem Urquell hin. Wo dasselbe zu Zwecken gebraucht wird, die nicht in ihm selbst liegen, da kann Wissenschaft vorhanden sein, aber ihr Geist ist wenigstens alsdann nicht lebendig.“

Das Prinzip zweckfreier Wissenschaft darf jedoch nicht darüber hinwegtäuschen, dass viele Gelehrte in der preußischen Universitätsreform gleichzeitig ein Bündnis mit dem absolutistischen Staat eingehen und somit nah am Pol weltlicher Macht stehen. Vor allem Johann Gottlieb Fichte ${ }^{8}$, aber auch Johann Jakob Engel ${ }^{9}$ und Friedrich Carl von Savigny ${ }^{10}$ verpflichten die neu gegründete Universität darauf, nicht nur eine private Bildungsanstalt zu sein, sondern auch die Ausbildung von Staatsdienern zu übernehmen. Diese Verantwortung, auf die vor dem Hinter-

7 Welche untergeordnete Rolle die Naturwissenschaften, immerhin Teil der philosophischen Fakultät, für die Universitätsreformer spielen, wird deutlich wenn Fichte (1910[1807]: 36) sie in seinem Deducirten Plan einer zu Berlin errichtenden höhern Lehranstalt mit einem Federstreich zu einem Teil der Geschichtswissenschaft erklärt und jede weitere Organisation einem noch zu bildenden Komitee überantwortet.

8 Johann Gottlieb Fichte (1762-1814), als Sohn eines Bandwebers geboren, wird zufällig vom Patronatsherrn als talentierter Schüler entdeckt und fortan auf dessen Kosten ausgebildet. Er studiert in Jena Theologie, hat das Studium aber nicht abgeschlossen, als er für neun Jahre an verschiedenen Stationen das Amt des Hauslehrers ausübt. Nachdem er bei Kant vorspricht und dieser zu seinem Fürsprecher wird, erhält Fichte 1794 eine Professur für Philosophie in Jena. Bereits nach wenigen Jahren wird er aber unter dem Vorwurf des Atheismus entlassen. Nach Privatvorlesungen in Berlin und einer weiteren Professur in Erlangen wird Fichte 1809 Dekan der philosophischen Fakultät an der neu errichteten Universität in Berlin. Nur ein Jahr später wird er zum ersten gewählten Rektor der Universität, bevor er 1814 überraschend stirbt (Boin 1989).

9 Johann Jakob Engel (1741-1802) studiert Theologie in Rostock und Bützow bevor er 1765 nach Leipzig geht und sich der Philosophie sowie den alten und neuen Sprachen zuwendet. Nach Tätigkeiten als freier Essayist und Theaterkritiker ist er Lehrer der Gebrüder Humboldt und des späteren Königs Friedrich Wilhelm III. Sieben Jahre lang leitet er das Königliche Theater in Berlin, dann nimmt er wieder schriftstellerische Tätigkeiten auf, verfasst Theaterstücke und versucht sich in der Formulierung einer allgemeinen Poetik. Schließlich wendet er sich auch populärphilosophischen Themen zu (Elschenbroich 1959).

10 Friedrich Carl von Savigny (1779-1861), früh verwaist und fortan von einem Assessor am Reichskammergericht erzogen, wird 1800 mit einem strafrechtlichen Thema in Marburg promoviert. Er schlägt die für einen Adligen ungewöhnliche Universitätslaufbahn ein und wird zum außerordentlichen Professor ernannt, schon bald erlangt er eine führende Stellung unter Juristen. Nach dreijährigen Forschungsreisen nimmt er einen Ruf nach Landshut an, 1810 wird er auf Empfehlung Humboldts nach Berlin berufen, wo er als Lehrer großen Einfluss ausübt. Die Zeit in Berlin ist durch die extensive Ausübung öffentlicher Ämter neben der wissenschaftlichen Tätigkeit gekennzeichnet. 1842 wird Savigny zum Staatsminister berufen, elf Jahre später beendet er seine wissenschaftlichen Aktivitäten (Nörr 2005). 
grund der Niederlage gegen Napoleon nicht selten mit patriotischem Nachdruck verwiesen wird (besonders Fichte 1910[1807]; Schleiermacher 1956[1808]; aber auch Steffens 1910[1809]; Humboldt 1956[1810]), trägt maßgeblich zur sozialen Legitimierung des Verständnisses einer zweckfreien Wissenschaft bei. Entscheidenden Rückhalt erhält das geisteswissenschaftliche Wissenschaftsideal auch durch die enge Verflechtung mit dem gebildeten Bürgertum. Gerade Professoren der philosophischen Fakultät erzielen mit Publikationen in Fächern wie Geschichte, Germanistik und Archäologie eine Wirkung, die weit über die Grenzen der Universität hinausgeht (Ringer 1987: 23-33; McClelland 1988: 37-38).

Der in der idealistischen Philosophie verankerte Wissenschaftsbegriff wird jedoch noch in der Reformphase von einer historisch-philologischen Häresie herausgefordert. Die Konfrontation ist vor allem von der Auseinandersetzung zwischen Idealismus und Historismus geprägt, die emblematisch von Georg Wilhelm Friedrich Hegel ${ }^{11}$ (1955[1822/1837]) und Leopold von Ranke ${ }^{12}$ (1975[1831/1832]) an der Berliner Universität ausgetragen wird. Konfliktlinien des Streits sind besonders die Methode der historischen Erkenntnis sowie der Zugang zum historischen Untersuchungsgegenstand. ${ }^{13}$ Die Abgrenzungsversuche gegenüber den

11 Georg Wilhelm Friedrich Hegel (1770-1831) stammt aus dem württembergischen Bildungsbürgertum. Der Musterschüler studiert bis 1793 Theologie in Tübingen, dort pflegt er eine enge Freundschaft zu Schelling und Hölderlin. Nach Tätigkeiten als Hofmeister und Hauslehrer habilitiert er sich 1801 in Jena, das durch Fichte geistiges Zentrum geworden ist. Die Kriegswirren verschlagen ihn nach Bamberg und Nürnberg, wo er Herausgeber der Bamberger Zeitung und Rektor eines Gymnasiums ist. Erst spät wird seine wissenschaftliche Leistung durch Berufungen anerkannt, die ihn zunächst nach Heidelberg und dann 1818 nach Berlin führen. Hier wird er, nach Jahren der Isolation in der Provinz, zum Mittelpunkt des geselligen Großstadtlebens. Vor allem aber erhält seine Philosophie nun äußersten wissenschaftlichen Einfluss. 1830 wird Hegel Rektor der Berliner Universität (vgl. Helferich 1989).

12 Leopold von Ranke (1795-1886) wächst in einem aufgeklärt-lutherischen Elternhaus auf und studiert in Leipzig klassische Philologie und Theologie. 1818 legt er in Berlin die Prüfung für das höhere Lehramt ab und wird Lehrer für Alte Sprachen und Geschichte an einem Gymnasium in Frankfurt/O. Sechs Jahre später begründet er mit seinem Erstlingswerk seine akademische Karriere und wird direkt nach Berlin berufen. Von Forschungsreisen nach Wien und Italien zurückgekehrt überträgt ihm die preußische Regierung die Leitung der Historisch-politischen Zeitschrift, bevor ihm Mitte der 1830er Jahre endgültig der historiographische Durchbruch gelingt. 1858 wird er Präsident der Historischen Kommission der Bayerischen Akademie der Wissenschaften (vgl. Muhlack 2003).

13 Hegel, der an der Universität Berlin auf dem Lehrstuhl von Fichte lehrt, verfügt über eine besondere Position im Feld. Bis zu seinem Tod 1831 vertritt er ein philosophisches System, das weit über die Grenzen Berlins hinaus die dominante geistige Strömung ist (vgl. den freilich recht tendenziösen Rückblick bei Virchow 1893: 15). Das hohe wissenschaftliche Prestige wird durch weltliche Macht ergänzt, da der preußische Kultusminister Karl vom Stein zum Altenstein den Philosophen persönlich an die Universität Berlin einlädt, was ihm den Ruf des preußischen Staatsphilosophen einbringt und seine Philosophie ,zu einer Art Hof- und Staatsphilosophie stempelt[e]“ (Hirschberger 1976: 433). Als Hegel 1818 auf den prestigeträchtigen Lehrstuhl berufen wird, hat sein Herausforderer Ranke gerade sein Studium der Theologie und Philologie beendet und 
spekulativen Elementen der idealistischen Geschichtsphilosophie und das Anliegen, empirische Fakten nicht durch die eigene Subjektivität zu verfälschen, sondern mit Hilfe einer systematischen Methode zu einer objektiven Erkenntnis zu gelangen, zeigen sich zwar besonders deutlich in der von Ranke begründeten Geschichtswissenschaft (zentral sind auch die Beiträge von Niebuhr 1811-1828 und Humboldt 1960[1821]). Ähnliche Tendenzen sind aber auch bei Friedrich August Wolf $^{14}$ (1999[1785/1789]) und Friedrich Schleiermacher ${ }^{15}$ (1977[1838]) auf dem Gebiet der philologischen Hermeneutik, in Karl Lachmanns $(1826,1827)$ historisch-kritischer Editionsarbeit deutscher Sprache und Literatur sowie in der historisch-dokumentierenden Leistung des Deutschen Wörterbuches der Gebrüder Grimm (1854-1971) für die Germanistik zu erkennen. Historische Durchdringung und Rekonstruktion sind zu dieser Zeit noch immer das Medium auf dem Weg zur eigenen Humanität, der antiken Welt wird nun aber auch ein genuiner, historischer Wert zugesprochen, der über den Genuss der Kunstwerke, über Sprachbeherrschung und Textverständnis hinausgeht.

tritt eine Anstellung als Gymnasiallehrer in Frankfurt/O. an. Er wird 1825 als außerordentlicher Professor für Geschichte an die Universität Berlin berufen. Hier hat Ranke wesentlichen Anteil an der Entwicklung der Geschichtsschreibung zu einer modernen Fachdisziplin, die in der Abgrenzung zur idealistischen Geschichtsphilosophie stattfindet. In Berlin angekommen fordert der dreißigjährige Ranke den etablierten Philosophen Hegel heraus, er kritisiert die „auf dem Weg der Spekulation zu ihren Resultaten gelangte[n] Philosophie, welche Ansprüche der Herrschaft über die Historie erhebt.“" Der spekulativen Erkenntnismethode der Hegelschen Geschichtsphilosophie, so Ranke weiter, ,entzieht sich und entweicht auf allen Seiten die Realität der Tatsache." (Ranke 1975[1831/1832]: 75) Auch Ranke gelingt die Verbindung hoher wissenschaftlicher und weltlicher Profite, und so verwundert es nicht, dass der Historiker schnell zur Hauptfigur des Historismus wird und auf eine überaus große Zahl von Schülern zurückblicken kann (Weber 1984: 200-217).

14 Friedrich August Wolf (1759-1824), als Sohn eines Schulmeisters und Kantors geboren, ist einer der ersten Studenten in Philologie bevor er, nach einer kurzen Tätigkeit als Gymnasiallehrer und Rektor einer Stadtschule, 1783 als ordentlicher Professor der Philologie und Pädagogik an die Universität Halle berufen wird. Ermuntert von Goethe entwickelt er hier eine rege literarische Tätigkeit, auch gründet er 1787 das philologische Seminar. 1805 wird er zum Geheimen Rat ernannt, 1807 wird er Mitglied der Preußischen Akademie der Wissenschaften, die ihn fünf Jahre später zu ihrem Ehrenmitglied ernennt. Wolf wird 1810 Professor an der Universität Berlin, an deren Errichtung er maßgeblichen Anteil hat (Jahn 2008).

15 Friedrich Daniel Ernst Schleiermacher (1768-1834), Sohn einer traditionsgebundenen Familie, die ihn für den Pfarrberuf vorgesehen hat, studiert Philosophie, Theologie und alte Sprachen in Halle. Als Prediger an der Charité in Berlin erhält er schnell Anschluss an den Kreis der Berliner Romantiker, zu denen auch die Brüder Humboldt gehören. Weiterhin lernt er hier Friedrich Schlegel kennen, der zeitlebens ein bedeutender Einfluss sein wird. Nach einer Professur für Theologie in Halle kehrt Schleiermacher 1807 nach Berlin zurück, trägt dort entscheidend zur Neuorganisation des Bildungswesens bei und wird 1810 zum Professor für Theologie berufen sowie 1811 zum Mitglied der Preußischen Akademie der Wissenschaften (Borkopp 1989). 


\subsubsection{Der Faktor Akteurspositionen}

Die eingangs beschriebene Krise der Universitäten am Ende des 18. Jahrhunderts schlägt sich auch in den charakteristischen Zuschreibungen der idealtypischen Akteurspositionen des Feldes nieder. Vor den Bildungsreformen ist die Lehre vertrocknet und erstarrt. Beobachter des Lehrbetriebs um 1800 beschreiben undisziplinierte und unkonzentrierte Studenten, die durch Übermut den Unterricht stören. Der Beitrag der wenig ambitionierten Professoren besteht in unmotivierten und steifen Kommentierungen autoritativer Lehrbücher, die eher Selbstgesprächen gleichen. Weil auch Prüfungen von niedrigem Niveau sind, ist von der Universität verliehenes kulturelles Kapital entwertet; es lässt sich nur eingeschränkt in welttliche Macht in Form von Anstellungen im Staatsdienst umwandeln und verspricht außerhalb der Universität nur geringe symbolische Profite. Besonders Professuren an der philosophischen Fakultät verfügen über ein geringes symbolisches Prestige, dienen sie doch häufig lediglich zum Aufstieg in eine der höheren Fakultäten. Die Schriften der Bildungsreformer zeigen, dass auch sie dieses Gesamtbild vor Augen haben (z. B. Schleiermacher 1956[1808]: 251, 275-276, 287; Fichte 1905[1811]: 36-37). Mit ihren Programmschriften haben sie es sich zur Aufgabe gemacht, diese bedrückende Lage zu verändern.

Um die Jahrhundertwende verändert sich die Wahrnehmung der Akteurspositionen des Professors und des Studenten grundlegend. Akademiker wachsen in eine dominante Stellung im sozialen Raum hinein, die vor allem auf ihrem kulturellen Kapital beruht (Baumgarten 2001: 108; Klinge 2004: 120). Wie gezeigt wurde, hält sich das quantitative Wachstum der geisteswissenschaftlichen Professuren noch in Grenzen; das Kollegium bleibt vorerst klein, weil der zunehmende Lehrerbedarf durch schlecht bezahlte Extraordinarien gedeckt wird. Wissenschaftliches Prestige wird daher vor allem den wenigen Ordinarien zugeschrieben, und so ist die für den Bildungsdiskurs entscheidende Figur im wissenschaftlichen Betrieb uneingeschränkt der Professor (vgl. Titze 1995: 66).

Zudem verstärkt sich diese Zuschreibungspraxis, weil sich auch die Dauer und der Bereich der fachlichen Zuständigkeit verändern. In der Vergangenheit hatten Professoren die oft sehr allgemein denominierten Lehrstühle nur wenige Jahre inne. Dazu war es üblich, frei werdende und der Sache nach benachbarte Lehraufträge zu übernehmen. So waren weder regelmäßige Fachwechsel noch längere Listen fachlicher Zuständigkeiten eine Seltenheit. Um die Jahrhundertwende entwickeln sich dann jedoch lebenslange Bindungen an ein (in der Regel allerdings noch immer recht umfassend definiertes) Kernfach. Lehrstühle werden nun auf Lebensdauer besetzt und Lehraufträge nicht mehr kumuliert. Insgesamt findet auf der Ebene des Rollenverständnisses die Entsprechung der fachlichen Differenzierung 
statt (Stichweh 1999: 341-342). Bevor die Spezialisierung dann so weit fortschreitet, dass der Umfang eines akademischen Faches unmöglich von einer einzigen Professur abgedeckt werden kann, ist im ersten Drittel des 19. Jahrhunderts eine Übereinstimmung von Fach und Professur möglich. Professoren vertreten nun in der Regel lebenslang die komplette Bandbreite ihres Faches, symbolisches Kapital wird nun an ihrer individuellen Position festgemacht. Blanke (1989: 356-358; vgl. Jarausch 2004: 303-309) zeigt außerdem, wie etwa ab den 1830er Jahren eine Professionalisierung stattfindet, bei der formale Studienabschlüsse wie die Promotion und die Habilitation wichtiger werden als Verwandtschaftsbeziehungen oder kollegiales Verhalten. Durch die drei Faktoren Professionalisierung, Fokussierung fachlicher Zuständigkeiten und enge Lehrstuhlhierarchie entsteht eine Konzentration wissenschaftlichen Prestiges auf die Akteursposition des gelehrten Professors.

Zeitgleich vereint diese Position die Rolle des Lehrers und des Forschers. Im 18. Jahrhundert ist die übliche Form akademischen Unterrichts noch das Vorlesen eines Kanons von Büchern, der nicht durch persönliche Ansichten des Lehrenden kontaminiert werden soll. Kant geht dann dazu über, neben den Büchern anderer Professoren auch seine eigenen, nicht selten ebenfalls in Buchform gefassten Positionen vorzutragen (Stark 1992: 549). Bereits bei der nachfolgenden Generation von Gelehrten, beispielsweise bei Fichte oder Hegel, findet dann die Entwicklung schulbildender Bücher unmittelbar im akademischen Unterricht statt. Bei dieser in die Praxis umgesetzten Einheit von Forschung und Lehre werden Studenten zu Teilhabern des intellektuellen Vorhabens ihrer Professoren (Stichweh 1999: 343-344).

Das Zusammenspiel zwischen Professor und Studenten, die als kreatives Element und als Kommunikationspartner dienen, ist mit einer immensen Steigerung der Ansprüche an Studenten verbunden (Fichte 1910[1807]: 9-10; Humboldt 1956[1810]: 381; s. a. Schelsky 1971: 75). Auch bei ihnen findet eine Neuinterpretation der akademischen Lebensform statt. Das Studentenleben wird nicht mehr als Gegenentwurf zu dem Erwachsenenleben begriffen, sondern als Vorbereitung auf ein verantwortliches Leben als Bürger. Im Rahmen von Selbstintegration und -disziplinierung werden Arbeits- und Leistungsethos sowie Bildungsziele der bürgerlichen Gesellschaft übernommen. Wichtig wird jetzt eine Selbstentfaltung mit Hilfe literarisch-ästhetischer oder philosophischer Inhalte, die weitgehend kohärent mit dem von den Universitätsreformern vertretenen neuhumanistisch-idealistischen Bildungsbegriff ist. Das Studium wird so zu einer prägenden Zeit, in der die gelehrte Lebensform als Habitus eingeübt wird (Brandt 2001: 132-133; Hardtwig 1985). Entscheidend ist jedoch, dass in der Konstruktion der Akteursposition des Studenten keine weiteren Sozialisierungs- oder Orientierungsbedürfnisse angelegt sind. Der Student wird zum Benutzer einer Anstalt, der Erziehungsauftrag 
wird mit der Berliner Reform von der Universität abgekoppelt und privaten Aktivitäten überlassen. In den so entstehenden Freiräumen bilden sich „spezifische Formen jugendlicher Selbsterziehung" (Brandt 2001: 147) aus, die in der Restaurations- und Vormärzzeit zu einer Blüte von Burschenschaften mit patriotischer, romantischer oder freiheitlicher Gesinnung führen.

Das affirmative Selbstverständnis der Studentengenerationen von 1750 bis 1819 sollte nicht darüber hinwegtäuschen, dass die Gelehrtengemeinschaft als gemeinsame Lebensform von Studenten und Professoren in erster Linie eine diskursive Zuschreibung ist. Hardtwig (1985: 176) beschreibt zwar, wie sich das „Generationenverhältnis $[\ldots]$ zu einem dynamischen Nacheinander im Zeichen der Fortschrittserwartung" wandelt, doch Humboldt ist sich sehr wohl darüber bewusst, dass dieser Idealvorstellung weder alle Studenten noch alle Professoren entsprechen können. Er versteht seinen Entwurf daher als normatives Leitbild, „und es braucht nur selten und nur hier und da wahrhaft hervorzutreten, um weit umher und lange nachher zu wirken“ (Humboldt 1956[1810]: 380; siehe dazu auch Fichte 1845[1794]: 329). In der Tat ist zu unterscheiden zwischen einem inneren Kreis der Studenten, der sich einem Professor anschließt, intensiv studiert und in der Regel selbst Wissenschaftler wird, und einer weitaus größeren Zahl von Studenten, die das Studium weniger ernst nehmen oder schlicht überfordert sind (McClelland 1980: 133-134; Brandt 2001: 144). Entscheidend ist jedoch, dass beide Akteurspositionen in der Lern- und Forschungstätigkeit als weitgehend gleichberechtigt wahrgenommen werden. Den Zuschreibungen der am Diskurs beteiligten Akteure zufolge sind Studenten und Professoren verbunden durch ihren Drang zur Erkenntnis und ihre gemeinsame Wahrheitssuche, die sicher nicht zufällig an einen sokratischen Dialog erinnert (Fichte 1910[1807]: 109; Humboldt 1956[1810]: 377).

Welche Annahmen über die symbolische Struktur des Feldes zur Zeit der preußischen Universitätsreform lassen sich angesichts dieser Ausführungen machen? Das Wissenschaftsverständnis ist zunächst von Universalismus und Zweckfreiheit geprägt. Diese Interpretation wird repräsentiert von der idealistischen Philosophie, die als orthodoxe Lehre des Feldes über besonders hohes symbolisches Kapital verfügt. Schnell wird diese Stellung vom Historismus herausgefordert, der eine stärkere Fakten- und Methodenorientierung verkörpert und damit die symbolische Ordnung des Feldes umdefiniert. Diese Auseinandersetzung kann als Übertragung des aufkommenden modernen Wissenschaftsverständnisses in die Logik des geisteswissenschaftlichen Feldes verstanden werden. Durch die Beamtenausbildung genießen geisteswissenschaftliche Fächer eine hohe soziale Legitimität, die jedoch ebenfalls nicht durch einen Bruch mit der spezifischen Logik des Feldes erkauft werden muss. Auch hier zeigt sich ein relativ hoher Autonomiegrad 
des Feldes, weil externe Anforderungen in die feldinterne Logik übersetzt werden. Schließlich lässt auch das Wissen über fachliche Kompetenz, Professionalität und Integration der Akteurspositionen auf die Ausbildung einer autonomen, feldeigenen Logik schließen. Die bereits für die materielle Struktur festgestellte Geschlossenheit der Struktur wiederholt sich bei den Professoren mit der starken Konzentration wissenschaftlichen Prestiges auf die Spitze einer steilen Hierarchie bei zugleich fortschreitender Spezialisierung.

\subsection{Die Phänomenstruktur des Bildungsdiskurses ${ }^{16}$}

\subsubsection{Ontologische Dimension}

Ein besonders dominantes, sich durch alle Ausdeutungen geisteswissenschaftlicher Bildung ziehendes Motiv ist die Freiheit von externen Anforderungen oder Einflüssen. Für Schleiermacher (1956[1808]: 263), der in dieser Frage eine der radikalsten und konsequentesten Positionen vertritt, tragen Gelehrte und Wissenschaftler ihre eigenen Gesetze in sich und benötigen daher weder Motivation noch Vorschriften von außen. Alles was zum wissenschaftlichen Geschäft der Universität gerechnet werden kann, muss ihm zufolge von den Wissenschaftlern selbst organisiert und gebildet werden. Auch für Fichte (1910[1807]: 17) beeinträchtigen Ablenkungen durch tägliche Bedürfnisse oder Nebenarbeiten den Charakter des Gelehrten und sollten daher vermieden werden. Die bereits bei Schleiermacher (1956[1808]: 279) artikulierte Sorge um den störenden Einfluss sozialer

16 Die Analyse der inhaltlichen Ebene des Bildungsdiskurses der preußischen Universitätsreform orientiert sich an den wichtigsten Äußerungen im geisteswissenschaftlichen Feld. Zu Illustrationszwecken werden die verschiedenen Elemente der fünf Dimensionen zu je einem Grundmotiv verdichtet, zu dem sich die einzelnen Beiträge dann unterschiedlich positionieren. Im empirischen Material sind die meisten Motive jedoch in unterschiedlichen Ausformulierungen auf allen Dimensionen vertreten.

Es ist bekannt, dass Humboldts Denkschrift Über die innere und äußere Organisation der höheren wissenschaftlichen Anstalten in Berlin (1956[1810]) erst etwa 90 Jahre nach seiner Tätigkeit im preußischen Innenministerium publiziert wird. Eine öffentliche Rezeption dieses Textes zum Zeitpunkt der preußischen Bildungsreformen hat daher nicht stattgefunden. Dennoch repräsentiert der Text, neben anderen zu Beginn des 19. Jahrhunderts verfassten Schriften von Humboldt, die Position des Autors und reflektiert darüber hinaus den damaligen Diskussionsstand. Gleiches gilt für Fichtes Deducirten Plan einer zu Berlin errichtenden höhern Lehranstalt (1910[1807]), der zwar 1807 verfasst, aber zu diesem Zeitpunkt vertraulich an von Beyme weitergegeben und, wiewohl er zuvor Humboldt zugänglich ist, erst zehn Jahre später aus dem Nachlass publiziert wird. Nichtsdestotrotz kann der Plan nicht nur als „,Dokument für den Geist einer Zeit“" (Spranger 1910: XXX), sondern vor allem als Repräsentation der Position Fichtes und seiner Sicht auf die Diskussion gesehen werden. Werden solche spezifischen Publikationsund Rezeptionsgeschichten einzelner Texte reflektiert, können sie daher als Stellvertretertexte für die Position ihrer Verfasser und für die zu diesem Zeitpunkt gegebene Diskurskonstellation verwendet und in das Korpus aufgenommen werden. 
Schichtungsmerkmale taucht bei Fichte (1910[1807]: 48-49) in dem Vorschlag auf, Uniformen zu tragen, um den Einfluss universitätsexterner Distinktionsmerkmale zu minimieren. „Jede äußere Gewalt“ ist, so auch Henrik Steffens ${ }^{17}$ (1910[1809]: 253), zu entfernen, ,,damit die emporstrebende Seele zum innern Verständnis gelange." Als letzte Deutung dieses Motivs ist Humboldts (1956[1810]: 377) Wort von „Einsamkeit und Freiheit“ zu nennen, das noch heute über eine bemerkenswerte symbolische Strahlkraft verfügt.

Diese Konstruktion geisteswissenschaftlicher Bildung als völlig frei von externen Einflüssen und Restriktionen, also die Annahme, dass Bildung einfach ungestört stattfinden muss, um ihre größte Wirkung zu entfalten, ist zur Zeit der preußischen Universitätsreform die deutlichste Ausprägung auf der ontologischen Dimension. Die Freiheit von äußeren Zwängen ist verbunden mit einer neuhumanistisch geprägten Idee der charakterlichen Veredelung, die im Bildungsprozess möglichst ungestört stattfinden soll.

\subsubsection{Funktionale Dimension}

Der Studienzeit kommt eine besondere Funktion bei der Charakterbildung zu, weil sie als die entscheidende Lebensphase für die Entwicklung von Talenten und Präferenzen verstanden wird. Steffens (1910[1809]: 233) und Schleiermacher (1956[1808]: 276-277, 301) begründen genau damit die Freiheit, in der wissenschaftliche Bildung stattfinden müsse. An der Universität werde der Student bewusst äußeren Einflüssen entzogen, um, frei von externen Störungen, vollkommen aus sich selbst heraus eine Persönlichkeit zu entwickeln. Nicht selten wird die Entfaltung der Persönlichkeit zum einzigen Zweck wissenschaftlicher Bildung erklärt, in jedem Fall ist sie anderen Bildungszielen wie einer zielgerichteten beruflichen Ausbildung symbolisch überlegen.

Die Vorstellung, dass Bildung aus der Wirkung des Bildungsgegenstands in den Tiefen des menschlichen Geistes heraus entsteht, findet sich gerade bei Humboldt (1956[1793]: 16) besonders deutlich ausformuliert: „Der Auffassende muß sich immer dem auf gewisse Weise ähnlich machen, das er auffassen will.“ Die Verbindung von Bildungsgegenstand und Charakter des sich Bildenden ist wech-

17 Henrik Steffens (1773-1845) studiert als Sohn eines Barbiers und Chirurgen in Kopenhagen Naturwissenschaften und später Medizin. 1796 setzt er das Studium in Kiel fort. Neben Kant, Jacobi und Fichte wird er vor allem von Friedrich Schelling geprägt, den er 1798 in Jena hört. Steffens wird 1804 als Professor für Naturphilosophie, Mineralogie und Physiologie nach Halle berufen, 1811 entkommt er einer Verhaftung wegen seines patriotischen Engagements durch die Annahme eines Rufes nach Breslau, wo er 1821/22 und 1829/30 zum Rektor ernannt wird. Mittlerweile genießt Steffens auch in Dänemark wissenschaftliche Anerkennung. 1832 wird er nach Berlin berufen und zwei Jahre später zum Rektor der Universität gewählt (Engelhardt 2008). 
selseitig konzipiert. Es geht also bei der Bildung auch darum, den Gegenstand so zu formen, dass dieser sich dem Geist annähere. Doch nicht nur Humboldt ( s. a. 1960[1793]: 234-237, 1956[1810]: 379, 1960[1821]: 588) vertritt diese neuhumanistische Position. Vor dem Hintergrund einer Wechselwirkung zwischen Charakter und Bildungsobjekt kann auch Hegels Phänomenologie des Geistes als Abhandlung zu einem neuhumanistischen Bildungskonzept gelesen werden. Der Bildungsprozess wird hier als ein Pfad des Zweifels verstanden, auf dem das Selbst wandelt. „Die Reihe seiner Gestaltungen, welche das Bewußtsein auf diesem Wege durchläuft, ist vielmehr die ausführliche Geschichte der Bildung des Bewußtseins selbst zur Wissenschaft.“ (Hegel 1907[1807]: 54) Die Dialektik wird dann zur Überwindung eines Widerspruches zwischen dem Bewusstsein und einem äußeren Objekt, in deren Rahmen sich beide Elemente einander angleichen können (vgl. Hegel 1907[1807]: 50-61; s. a. Smith 1988). Auch Fichte macht es zur Bestimmung des Gelehrten (1845[1794]: 298), „die Dinge [...] zu modificiren, und sie selbst zur Übereinstimmung mit der reinen Form seines Ich zu bringen, damit nun auch die Vorstellung von ihnen [...] mit jener Form übereinstimme." Wie Humboldt scheint es auch ihm in der wissenschaftlichen Bildung vor allem um eine „sittliche Veredlung des ganzen Menschen“ (Fichte 1845[1794]: 332, vgl. 301) zu gehen. Fichte vertritt ansonsten jedoch einen republikanischen Bildungsbegriff, er begreift wissenschaftliche Bildung stärker als staatliches Projekt und hat somit immer auch die Ausbildung zum Staatsbürger und -diener im Blick. Dass er dennoch jede Wissenschaft, die nicht zuvorderst Humanität und kulturelle Bildung in den Studenten weckt, für nichtig erklärt, unterstreicht das symbolische Gewicht, das der Diskurs neuhumanistischen Bezügen verleiht. Fichte (1845[1794]: 328, 330) führt diese Ausdeutung jedoch wieder mit der für ihn charakteristischen Kopplung von wissenschaftlicher Bildung und staatlichen Interessen zusammen. Die erworbene Bildung findet ihren letzten Zweck für Fichte demnach nicht in der Veredelung des menschlichen Charakters um ihrer selbst Willen, vielmehr muss sie zum allgemeinen Nutzen der Gesellschaft angewendet werden. Diesem Argumentationsmuster folgen auch Friedrich August Wolf (1990[1807]: 46) und Steffens (1910[1809]: 243).

Trotz der von Steffens, Wolf und Fichte vorgenommenen Verbindung zwischen allgemeiner Charakterbildung und den besonderen Interessen des Staates scheint im Bildungsdiskurs eine neuhumanistisch-idealistische Orthodoxie zu verfügen, dass alle Studenten ihre Zeit an der Universität mit dem Studium eines universellen Wissensgefüges beginnen sollten. 


\subsubsection{Objektdimension}

Diese Vorstellung einer universellen Wissensordnung wird verstanden als ideale Grundlage für eine dezidiert wissenschaftliche Bildung, in der es darum geht, in jedem einzelnen die Idee wissenschaftlichen Erkennens zu entfachen und als den Menschen leitendes Prinzip zu erwecken. Wird Bildung, wie es diese Ausdeutung auf der Objektdimension nahe legt, als Beschäftigung mit wissenschaftlichen Gegenständen verstanden, erhält die beispielsweise von Humboldt (1956[1810]: 379) hervorgehobene Veredelung des Charakters im Bildungsprozess eine besondere Akzentuierung: Weil Bildungs- und Forschungsgegenstand tendenziell zusammenfallen, liegt der bildende Effekt in der Annäherung von Charakter und dezidiert wissenschaftlichem Gegenstand (besonders deutlich artikuliert wird dies für klassisch-historische Inhalte, siehe Humboldt 1956[1793]: 26, 1960[1821]: 588). Zwar betont Humboldt (z. B. 2008[1814]: 921) die Bildung an klassischen Untersuchungsobjekten, doch er räumt auch ein, dass die Art und Weise der Wissensvermittlung den Vorrang vor dem konkreten Gegenstand der Bildung hat. Auch für Fichte (1910[1807]: 8) ist wissenschaftliche Bildung tendenziell vom Bildungsgegenstand unabhängig. Dabei steht diese von den Untersuchungsgegenständen unabhängige Entfaltung eines allgemeinen Forschungsgedankens für ihn keineswegs im Widerspruch zur Ausbildung fähiger Staatsdiener. Wie er positionieren sich auch Steffens (1910[1809]: 217, 267) und Schleiermacher (1956[1808]: 238). Letzterer unterstreicht das Primat des wissenschaftlichen Geistes vor jedem Untersuchungsobjekt, wenn er das Universitätsstudium als „Lernen des Lernens“ beschreibt. Erst wenn diese allgemeine wissenschaftliche Bildung erreicht sei, könne auf dieser Basis eine eigenständige Vertiefung in einen besonderen Forschungsbereich unternommen werden (Schleiermacher 1956[1808]: 242, 250). Nur Hegel (1955[1822/1837]: 287-288) betont, dass die Philosophie den Erwerb bestimmter Kenntnisse gewährleisten solle, und dass diese Aufgabe untrennbar mit bestimmten Bildungsgegenständen verbunden sei.

Der Gegenstand, an dem sich geisteswissenschaftliche Bildung vollzieht, fällt also mit dem Forschungsgegenstand zusammen. Weil aber bei der Ausbildung des wissenschaftlichen Habitus der konkrete Untersuchungsgegenstand tendenziell hinter die Vermittlung einer allgemeinen Haltung zurücktritt, kann sich Bildung in relativer Unabhängigkeit von einem konkreten Gegenstand vollziehen.

\subsubsection{Praktische Dimension}

Ein wichtiges Instrument der wissenschaftlichen Sozialisierung ist die Gemeinschaft von Studenten und Professoren. Studenten müssen sich, so die allgemeine Annahme, in ihrem wissenschaftlichen Streben vom Professor gefördert und in- 
spiriert fühlen (vgl. Humboldt 1956[1810]: 377, 382-383). Es entspricht den bis hier skizzierten Positionen, wenn vor allem Schleiermacher (1956[1808]: 263, 275) dieser Gelehrtengemeinschaft einen hohen Grad akademischer Freiheit zuschreibt, während Fichte (1845[1794]: 329-330, 1905[1811]: 22) vor dem Missbrauch dieser Freiheit durch die Studenten warnt und einen staatlich subventionierten Müßiggang fürchtet.

Eine weitere Ausdeutung auf der praktischen Dimension ist die Annahme, dass universitäre Bildung keine sozialen Vorbedingungen haben, sondern prinzipiell allen Studenten zugutekommen sollte - selbst wenn die Mehrzahl gar keine wissenschaftliche Karriere anstrebt oder sich in diesem freien System nicht zurechtfindet. Die Rhetorik des Bildungsdiskurses sieht für wissenschaftliche Bildung daher keine praktische Einschränkung auf Grundlage von Schichtzugehörigkeit vor. Wer die wissenschaftliche Erkenntnis suche, so nimmt beispielsweise Schleiermacher (1956[1808]: 276-278, 282) an, sei von vornherein sittlich und edel genug, um sie in den akademischen Freiräumen finden zu können. Trügen Studenten die Sittlichkeit nicht bereits in sich, eigneten sie sich ohnehin nicht für dieses Unterfangen und seien der wissenschaftlichen Bildung nicht fähig. Schleiermacher nimmt mit dieser radikal anmutenden Interpretation keineswegs eine Extremposition ein. Auch Humboldt (1964b[1809]: 218) will ausnahmslos jedem seine neuhumanistisch verstandene Bildung zukommen lassen: „Jeder ist offenbar nur dann ein guter Handwerker, Kaufmann, Soldat und Geschäftsmann, wenn er an sich und ohne Hinsicht auf seinen besondern Beruf ein guter, anständiger, seinem Stande nach aufgeklärter Mensch und Bürger ist." Lediglich Fichte (1845[1794]: 292) mahnt, dass die Studenten, denen der wissenschaftliche Geist fehle, ihrer Bestimmung in gewöhnlicheren Bereichen nachkommen, aber nicht die Gelehrtengemeinschaft durch Nutzenforderungen zu sich herabziehen sollten.

Festzuhalten ist hier, dass die Zugangsvoraussetzung zu wissenschaftlicher Bildung ein Habitus ist, dessen Ausbildung zwar zu jeder Zeit eng an praktische Voraussetzungen wie eine klassische Vorbildung auf dem Gymnasium gekoppelt bleibt. Gleichzeitig verbietet es die universalistisch-neuhumanistische Idee einer allgemeinen Bildung aber zumindest in den ersten Jahren des 19. Jahrhunderts, dass diese faktische Exklusion zu explizit formuliert wird oder über diese impliziten Mechanismen hinausgeht. Im Gegenteil finden sich sogar zahlreiche Referenzen auf einen explizit inklusiven Bildungsbegriff (vgl. Fichte 1845[1794]: 324, 1910[1807]: 20; Humboldt 1960[1793]: 236, 1964b[1809]: 217; Savigny 1990[1808]: 265). ${ }^{18}$

18 Das Ideal der nicht nur inhaltlich, sondern auch sozial allgemeinen Bildung als Vorbereitung für gleich welchen Beruf fällt, zusammen mit dem damit verwandten Ideal des frei und autonom 


\subsubsection{Subjektdimension}

Neben dem Professor und dem Studenten werden im geisteswissenschaftlichen Bildungsdiskurs der preußischen Universitätsreform vor allem zwei weitere verantwortliche Bildungssubjekte auf der entsprechenden Dimension angerufen: die Universität und der Staat.

Ist die Philosophie das disziplinäre Zentrum des allgemeinen Zusammenhangs des Wissens, so bildet die Reformuniversität das institutionelle Pendant. Steffens (1910[1809]: 217) unterstreicht, wie die Universität durch die ,innere Organisation alles Wissens“ zum „Mittelpunkt des Ganzen“ wird. Für Schleiermacher (1956[1808]: 249, vgl. 259) vereinigen Universitäten die Vielfalt der Fächer zu einem universellen Gefüge, das sie zum Mittelpunkt jedes wissenschaftlichen Unterfangens macht und die Durchsetzung „eines handwerksmäßigen Wesens und einer kläglichen Beschränktheit in allen Fächern" verhindert. Bei Humboldt äußert sich die Auffassung von der Universität als Zentrum aller wissenschaftlichen Unternehmung schließlich ganz praktisch darin, dass er dem König im Antrag auf Errichtung der Universität Berlin (1964a[1809]: 115) vorschlägt, alle bereits in Berlin vorhandenen Institute, Akademien und Sammlungen sowie die Bibliothek, das Observatorium und den botanischen Garten in ein organisatorisches und organisches Ganzes einzubinden.

In dieser von Schleiermacher und Humboldt besonders deutlich artikulierten Interpretation der Universität als organisatorischem Gerüst des universellen wissenschaftlichen Geistes wird bereits deutlich, dass Universitäten vor allem als Orte der Wissenschaft konzipiert sind. Als Subjekt im Bildungsdiskurs ist ihre Aufgabe die Erzeugung, Ausbildung und Beaufsichtigung des wissenschaftlichen Geistes in den Universitätsangehörigen (Schleiermacher 1956[1808]: 238; Humboldt 1956[1810]: 377-380). In dieser Funktion wird die Universität von der philosophischen Fakultät dominiert, an deren Spitze wiederum die Philosophie steht (Fichte 1845[1794]: 328, 1910[1807]: 26; Schleiermacher 1956[1808]: 239-241, 250, 260; Steffens 1910[1809]: 261; Hegel 1990[1816]: 289).

Die enge Beziehung zwischen Universität und Staat ist eine Besonderheit der untersuchten historischen Konstellation. Weil der Diskurs in das politische Projekt des Wiederaufstiegs Preußens eingebettet ist, erfordern die Zensurmechanismen des Feldes von allen Akteuren des Bildungsdiskurses eine Positionierung zum Einfluss des Staates auf geisteswissenschaftliche Bildung. Den dadurch auf-

denkenden Menschen, schon bald der nach 1815 einsetzenden Restauration zum Opfer. Die sich nun etablierende Unterscheidung zwischen höherer und niedrigerer Bildung privilegiert zwar die Stellung der Universität, bereitet aber auch ihrer ursprünglich diese Grenzen transzendierenden Bildungsidee ein Ende. Die soziale Herkunft des studentischen Bildungssubjekts wird nun auch diskursiv wieder zu einem relevanten Merkmal. 
gespannten argumentativen Möglichkeitsraum besetzen Schleiermacher auf der einen und Fichte auf der anderen Seite. Letzterer will mit seinem republikanischen Verständnis von Bildung die Universitäten dem Staat unterstellen, um sie zu dezidiert nationalen Bildungsanstalten zu machen. Typischerweise erklärt diese Rhetorik die akademische Freiheit zwar zum höchsten Gut der Universität, begründet sie aber vor allem mit der Hervorbringung fähiger Staatsbürger und -diener (Fichte 1910[1807]: 17, 18, 24, 1905[1811]: 22-23). Steffens folgt Fichte in seiner Interpretation der Rolle des Staates. Für ihn werden den Universitäten die „Jünglinge des Staates“ anvertraut, sie sind ,die Pfleger des nationalen Geistes“, die daher „,innig [...] mit dem Wesen deutscher Eigentümlichkeit verschmolzen sind“ - ein Verfall der Universität ist unter diesen Umständen unweigerlich mit einem Verfall der Nation verbunden (Steffens 1910[1809]: 247-248, vgl. 226-229).

Schleiermacher lehnt dagegen jeglichen staatlichen Zugriff auf die Universitäten ab und versteht sie als Schutzraum, in dem das wissenschaftliche Unterfangen die größtmögliche Freiheit genießen muss. Sein Hauptanliegen ist vor allem, „den allmählich vorherrschend gewordenen Einfluss des Staates wieder in seine natürlichen Grenzen zurückzuweisen, und dagegen immer mehr den Charakter des wissenschaftlichen Vereins in diesen ihm zunächst angehörigen Anstalten hervortreten zu lassen." (Schleiermacher 1956[1808]: 272, vgl. 232, 244-245, 249, 268-269, 270)

Eine Mittlerposition nimmt in dieser Konstellation Humboldt ein. Er folgt mit seinem neuhumanistischen Verständnis von Bildung zunächst eher Schleiermacher und macht dessen philosophische Überlegungen für den politischen Diskurs anschlussfähig (zum großen Einfluss Schleiermachers auf Humboldt siehe Rüegg 1997). Generell betrachtet auch Humboldt die Verwertungsansprüche des Staates als Gefahr für die wissenschaftliche Autonomie. Er schlägt dem König sogar vor, die neu zu gründende Universität über Domänen und Pfründe zu finanzieren, um sie unabhängig von den jährlichen Zuwendungen des Staates zu machen (Humboldt 1964a[1809]: 117; s. a. 1956[1810]: 378). Dieser Vorschlag wird jedoch abgelehnt, und auch Humboldt selbst überträgt den von Schleiermacher übernommenen Gedanken der konsequenten Autonomie vom Staat letztlich nur begrenzt auf die konkrete organisatorische Ebene. Im Allgemeinen konzediert er eine enge Beziehung zwischen dem Geschäft der Universität und den Bedürfnissen des Staates und folgt hier in der Tendenz Fichte (Humboldt 1956[1810]: 380, 384-385). ${ }^{19}$ In dieser argumentativen Zwiespältigkeit zeigt sich die Doppelrolle Humboldts als

19 Der Unterschied zu Fichte liegt nun darin, dass dieser die akademische Autonomie noch mit den Bedürfnissen des Staates legitimieren muss, während die wissenschaftliche Interessenlosigkeit für Humboldt als Letztbegründung fungiert. 
Staatsmann und Wissenschaftler. Der weltlichen und der wissenschaftlichen Logik folgend erklärt er die Freiheit der Wissenschaft zum obersten Prinzip der Universität, zu einem Prinzip jedoch, von dem letztlich auch der Staat profitiere und das zu wahren deshalb auch in seinem Interesse sei (Humboldt 1956[1810]: 378381, 2008[1814]: 920). Nur eine freie, vor Praxisanforderungen geschützte Universität sei für den Staat von Nutzen, weil die für die Belange des Staates wichtigen Kenntnisse und Absolventen für Humboldt nur in einem ungestörten Kontext produziert werden können. Damit ist eine weitgehend akzeptierte Sicht auf den Staat als verantwortliches Bildungssubjekt benannt, die zum einen wissenschaftliche Autonomie und ein ausgeprägtes Selbstbestimmungsrecht der Universitäten fordert, dies zum anderen aber nicht gegen die Bedürfnisse des Staates formulieren muss. Einer solchen Deutung der Beziehung von Staat und universitärer Wissenschaft folgen neben Humboldt auch andere Autoren der Debatte, zum Beispiel Engel (1990[1802]: 10) und Savigny (1990[1808]: 259-261).

Durch die je nach Ausdeutung mehr oder weniger enge Verknüpfung mit den weltlichen Interessen des Staates oder der Nation kann die beschriebene freiheitliche Interpretation des universitären Bildungssubjekts sowie der Wissenschaft im Allgemeinen zumindest zeitweise auch über die Grenzen des universitären Feldes hinaus Legitimität beanspruchen.

Nach der inhaltlichen Analyse des Bildungsdiskurses ergibt eine Aggregation der unterschiedlichen Positionen zu einer einzigen (und der Annahme nach dominanten) Interpretation geisteswissenschaftlicher Bildung folgende diskursive Phänomenstruktur. Die Deutung von Bildung als Selbstbestimmung des zur autonomen Selbsttätigkeit fähigen, mündigen Menschen legt Zeugnis für den Einfluss des besonders von Kant, aber auch von Schleiermacher und Fichte in die Reform getragenen Idealismus ab. Die Bildung an zumeist klassischen Gegenständen ist wiederum maßgeblich auf den Einfluss des Neuhumanismus zurückzuführen, den im Bildungsdiskurs beispielsweise Humboldt repräsentiert. Es geht hier um die harmonische Kultivierung der individuellen Anlagen zu einer universell gebildeten Persönlichkeit, mit der die Idee der Menschheit im Einzelnen entfaltet wird. Diese Humanität ist der letzte Zweck, vor dem auch konkrete Bildungsgegenstände zurücktreten. Die soziale Form dieser Ausdeutung von Bildung ist die Gelehrtengemeinschaft, in der Lehrer und Schüler gemeinsam nach der reinen Erkenntnis streben. Auch die Annahme, dass diese Bildung allen Menschen zugutekommen sollte, kann dem Neuhumanismus zugerechnet werden. Der Ort dieser Bildungsidee ist schließlich die Universität, in der die philosophische Fakultät den Überbau des universellen Wissens repräsentiert. Sie wird geschützt von einem Staat, 
dem ein Interesse daran zugeschrieben wird, dass die so definierte Bildung seinen Bürgern und Beamten zukommt.

\section{Die wechselseitige Ergänzung von Feld- und Diskursanalyse}

Dieser Entwurf einer wissenssoziologischen Feld- und Diskursanalyse hat erstens zum Ziel, ein genaueres Verständnis des geisteswissenschaftlichen Bildungsdiskurses zur Zeit der preußischen Universitätsreform zu ermöglichen. Erst der genaue Blick auf den Diskurszusammenhang und die Relationierung mit seinem Produktions- und Rezeptionskontext erhellt die spezifischen Umstände und Inhalte, aus denen sich das Selbstverständnis der Geisteswissenschaften trotz aller Umbrüche im universitären Feld noch heute speist. Der historische Referenzpunkt macht zum einen klar, welcher Statusverlust für die Geisteswissenschaften mit dem Erstarken der universitären Naturwissenschaften im weiteren Verlauf des 19. Jahrhunderts einhergeht. Der nun entstehende epistemologische, methodologische und methodische Begründungs- und Rechtfertigungsdruck sowie die veränderten Anforderungen und Ansprüche seitens der Gesellschaft schlagen sich ab dem letzten Drittel des 19. Jahrhunderts deutlich in den bis dahin eher selbstreferentiellen Reflektionen nieder. Zum anderen lässt sich nun die Spannung erahnen, die zwischen dem im beschriebenen Bildungsdiskurs wurzelnden geisteswissenschaftlichen Selbstverständnis und der tief greifenden Umwälzung der Strukturen des Feldes im Rahmen der Bildungsexpansion ab den sechziger Jahren des 20. Jahrhunderts entstanden ist. Die weitgehende Öffnung materieller und symbolischer Strukturen entzieht der traditionellen Identität der Geisteswissenschaften die Legitimationsgrundlage und evoziert nicht zuletzt ein besonders ausgeprägtes Krisenempfinden (vgl. Hamann 2009, 2011; s. a. Münch 2009: 169-172).

Zweitens soll neben den Anregungen, die aus der Analyse des empirischen Gegenstands hervorgehen können, gezeigt werden, dass Feldanalyse und Diskursanalyse das Potential haben, komplementär den Ergänzungsbedarf des jeweils anderen Ansatzes zu adressieren. Werden beide Perspektiven produktiv aufeinander bezogen dann wird deutlich, wie der soziale Kontext die diskursive Produktion und Rezeption von Wissen beeinflusst. Ähnlich einer sozialen Tatsache kann er Zwänge ausüben, Spannungen erzeugen oder Druck bewirken, aber auch Möglichkeitsräume eröffnen, in denen die Wissensproduktion und -rezeption relativ autonom stattfinden kann. Zugleich erlaubt eine inhaltliche Analyse dieser Wissensordnungen den detaillierten Blick auf eine Strukturierungsebene unterhalb von Stilen, Genres und Disziplinen. Weil diese Ebene den Kontext nicht einfach abbildet oder durch ihn 
determiniert ist, wird ihr ein eigenständiger Platz im Analyserahmen eingeräumt. Abschließend gilt es, diese beiden Fäden aufzunehmen und zusammenzuführen.

Für die materielle Dimension wurde gezeigt, dass sich die Verteilungsstruktur von Ressourcen quantitativ leicht öffnet und qualitativ weitgehend geschlossen bleibt. Das bedeutet, dass von einer offeneren Verteilung der Ressourcen vor allem bereits begünstigte Positionen profitieren. So sorgt der geringe Differenzierungsgrad der institutionellen Ebene für ein enges disziplinäres Profil und eine relative Schließung der Wissensproduktion, da sich Vertreter neuer Schulen und externe Einflüsse unter diesen Umständen nur schwer durchsetzen können. Selbst der Ausbau der Kerndisziplinen kann hier als Stärkung der herrschenden Lehre verstanden werden und zeitigt daher Schließungseffekte. Die Analyse der Population des Feldes legt eine ähnliche Struktur nahe. Zwar ist sowohl für Studenten wie auch für Professoren ein leichter Zuwachs zu verzeichnen. Weil davon aber vor allem das Bildungsbürgertum profitiert, bleibt die Verteilungsstruktur qualitativ geschlossen.

Die Untersuchung der symbolischen Dimension zeigt, dass auch die Bewertungsstruktur nicht völlig geschlossen ist. Wie bei der materiellen Struktur ist die leichte Öffnung jedoch nicht qualitativer Natur, bleibt die (Neu-)Bewertung von Ressourcen doch nur bereits etablierten Positionen vorbehalten. Die Auseinandersetzung zwischen philosophisch-idealistischem und historisch-historistischem Wissenschaftsverständnis zeigt zwar, dass die symbolische Ordnung keineswegs unumstritten ist. Neben diesen dominanten Ausdeutungen legitimer Wissenschaft gibt es aber keine weiteren Interpretationsvorschläge, die in ähnlicher Weise konkurrenzfähig wären. Auch die Konzentration wissenschaftlichen Prestiges auf die Position des gelehrten Professors weist auf eine enge symbolische Hierarchie hin, an deren Spitze ein Deutungsoligopol bereits herrschender Akteure steht. Für die symbolische Struktur ist daher ebenfalls eine lediglich quantitative Öffnung zu erkennen, die eine Umstrukturierung nur von bereits dominanten Positionen aus erlaubt.

Die in der Analyse des Bildungsdiskurses skizzierten Deutungen scheinen dieser grundsätzlichen Struktur in vielerlei Hinsicht auf einer eigenen Ebene zu entsprechen. Die Untersuchung legt nahe, dass bestimmte diskursive Strukturierungen eines Bildungsbegriffes ihre Voraussetzung in einer spezifischen Feldstruktur haben. Beispielsweise erfordert das neuhumanistisch-idealistische Bildungsverständnis konkrete soziale Bedingungen wie eine klassische Vorbildung in der Schule, das in der Gelehrtengemeinschaft realisierte Betreuungsverhältnis oder die nach dem Studium typischen Karrierewege. Weiterhin lässt sich die starke Prägung des Bildungsbegriffs durch Neuhumanismus und Idealismus auch auf die kompakte Fächerstruktur und das institutionelle Gewicht von Kerndisziplinen zurückführen, die genau dieses Profil aufweisen. 
Anders als Bourdieu (2006: 293) nahe zu legen scheint, sind diskursive Äußerungen jedoch nicht lediglich „Resultanten“ einer objektiven Struktur, sondern erweisen sich als Ebene mit eigenen Beharrungskräften, einem relativ autonomen Strukturierungsvermögen sowie eigenem Erkenntnispotential. So ermöglicht erst eine detailliertere Analyse des Bildungsdiskurses ein Verständnis der Beziehung zwischen geisteswissenschaftlicher Bildung und Staat. Zwar lässt die politische Einbindung der Gelehrten des Bildungsdiskurses in den Reformprozess bereits einen Einfluss staatlicher Interessen vermuten. Wie genau sich der enge Bezug zum weltlichen Herrschaftspol dann aber artikuliert, und dass sich dieser Bezug keineswegs in einer Aufgabe der feldinternen Logik, sondern gerade in der Übersetzung staatlicher Interessen in diese vollzieht, kann erst eine Analyse der inhaltlichen Ebene zeigen. Diskursive Beharrungskräfte zeigen sich beispielsweise darin, dass der Bildungsdiskurs zunächst weitgehend unberührt von den Auseinandersetzungen zwischen Idealismus und Historismus bleibt. Eine direkte Übersetzung der Feldstrukturen in diskursive Strukturen würde eine frühere und deutlichere Resonanz der historistischen Häresie im Bildungsdiskurs vermuten lassen. Hier zeigt sich jedoch eine an den Mechanismus der Hysteresis erinnernde Trägheit diskursiver Strukturen. Schließlich drückt sich das eigene Strukturierungsvermögen der diskursiven Ebene darin aus, dass die soziale Exklusivität, die sich in der Struktur der Population zeigt und die das geisteswissenschaftliche Bildungsverständnis faktisch einfordert, keineswegs auch so artikuliert werden kann, weil sonst ein Widerspruch zum neuhumanistischen Bildungsbegriff entstünde. Der eher inklusive Bildungsbegriff der Neuhumanisten steht in einem Spannungsverhältnis zu der im Feld stattfindenden Exklusion und kann daher schwerlich als Manifestation der objektiven Feldstrukturen verstanden werden. Hier wird am konkreten Beispiel deutlich, dass die Analyse der diskursiven Wissensproduktion eine produktive Ergänzung der Feldanalyse darstellt, weil sie eine relativ eigenständige Strukturierungsebene freilegt, die sich gerade nicht aus der Kontextstruktur ableiten lässt, sondern über eine eigene Logik verfügt. 


\section{Literatur}

Adorno, Theodor W. (2006[1959]): Theorie der Halbbildung. Frankfurt am Main: Suhrkamp Angermüller, Johannes (2007): Nach dem Strukturalismus. Theoriediskurs und intellektuelles Feld in Frankreich. Bielefeld: transcript

Angermüller, Johannes/Maeße, Jens/Standke, Jan (Hrsg.) (2011): Moving (Con)Texts. Produktion und Verbreitung von Ideen in der globalen Wissensökonomie. Berlin: Peter Lang. Im Erscheinen

Anrich, Ernst (Hrsg.) (1956): Die Idee der deutschen Universität. Die fünf Grundschriften aus der Zeit ihrer Neubegründung durch klassischen Idealismus und romantischen Realismus. Darmstadt: Wissenschaftliche Buchgesellschaft

Bachmaier, Helmut/Fischer, Ernst Peter (Hrsg.) (1997): Der Streit der Fakultäten. Oder die Idee der Universität. Konstanz: Universitätsverlag Konstanz

Baumgarten, Marita (1997): Professoren und Universitäten im 19. Jahrhundert: zur Sozialgeschichte deutscher Geistes- und Naturwissenschaftler. Göttingen: Vandenhoeck \& Ruprecht

Baumgarten, Marita (2001): Professoren und Universitätsprofile im Humboldt‘schen Modell 18101914. In: Schwinges (2001): 105-129

Blanke, Horst Walter (1989): Historiker als Beruf. Die Herausbildung des Karrieremusters, Geschichtswissenschaftler' an den deutschen Universitäten von der Aufklärung bis zum klassischen Historismus. In: Jeismann (1989): 343-360

Boin, Manfred (1989): Fichte, Johann Gottlieb. In: Lutz (1989): 239-243

Borkopp, Peter (1989): Schleiermacher, Friedrich Daniel Ernst. In: Lutz (1989): 702-705

Bourdieu, Pierre (1969): Intellectual field and creative project. In: Social Science Information 8. 2. 89-119

Bourdieu, Pierre (1988): Die politische Ontologie Martin Heideggers. Frankfurt am Main: Suhrkamp

Bourdieu, Pierre (1992): Homo academicus. Frankfurt am Main: Suhrkamp

Bourdieu, Pierre (1993a): Die Zensur. In: Bourdieu (1993b): 131-135

Bourdieu, Pierre (1993b): Soziologische Fragen. Frankfurt am Main: Suhrkamp

Bourdieu, Pierre (1998a): Praktische Vernunft. Zur Theorie des Handelns. Frankfurt am Main: Suhrkamp

Bourdieu, Pierre (1998b): Vom Gebrauch der Wissenschaft. Für eine klinische Soziologie des wissenschaftlichen Feldes. Konstanz: UVK

Bourdieu, Pierre (2001): Die Regeln der Kunst. Genese und Struktur des literarischen Feldes. Frankfurt am Main: Suhrkamp

Bourdieu, Pierre (2004): Science of Science and Reflexivity. Cambridge: Polity Press

Bourdieu, Pierre (2006): Die Praxis der reflexiven Anthropologie. In: Bourdieu et al. (2006): 251-294

Bourdieu, Pierre/Wacquant, Loïc D. (Hrsg.) (2006): Reflexive Anthropologie. Frankfurt am Main: Suhrkamp

Brandt, Harm-Hinrich (2001): Studierende im Humboldt'schen Modell des 19. Jahrhunderts. In: Schwinges (2001): 131-150

Bruch, Rüdiger vom (1999): Differenzierung und Professionalisierung. Von der Propädeutik zum Motor der modernen Forschungsuniversität. In: Schwinges (1999): 391-401

Diaz-Bone, Rainer (2002): Kulturwelt, Diskurs und Lebensstil. Eine diskurstheoretische Erweiterung der bourdieuschen Distinktionstheorie. Opladen: Leske + Budrich

Diemer, Alwin (1974): Geisteswissenschaften. In: Ritter (1974c): 212-215

Dreyfus, Hubert L./ Rabinow, Paul (1987): Michel Foucault. Jenseits von Strukturalismus und Hermeneutik. Frankfurt am Main: Athenäum

Elschenbroich, Adalberg (1959): Engel, Johann Jakob. In: Stolberg-Wernigerode et al. (1959): 504-505 
Engel, Johann Jakob (1990[1802]): Denkschrift über Begründung einer großen Lehranstalt in Berlin. In: Müller (1990): 6-17

Engelhardt, Dietrich von (2008): Steffens, Henrik. In: Vierhaus (2008): 626

Eulenburg, Franz (1904): Die Frequenz der deutschen Universitäten. Von ihrer Gründung bis zur Gegenwart. Leipzig: B. G. Teubner

Fichte, Immanuel Hermann (Hrsg.) (1845): Johann Gottlieb Fichtes sämmtliche Werke. Bd. 6. Berlin: Verlag von Veit und Comp

Fichte, Johann Gottlieb (1845[1794]): Einige Vorlesungen über die Bestimmung des Gelehrten. In: Fichte (1845): 291-346

Fichte, Johann Gottlieb (1905[1811]): Über die einzig mögliche Störung der Akademischen Freiheit. Heidelberg: Carl Winter's Universitätsbuchhandlung

Fichte, Johann Gottlieb (1910[1807]): Deducirter Plan einer zu Berlin zu errichtenden höhern Lehranstalt. In: Spranger (1910): 1-104

Flitner, Andreas (Hrsg.) (1956): Wilhelm von Humboldt. Schriften zur Anthropologie und zur Bildungslehre. Düsseldorf/München: Verlag Helmut Kupper

Flitner, Andreas/Giel, Klaus (Hrsg.) (1960): Wilhelm von Humboldt. Werke in fünf Bänden. Bd. I. Darmstadt: Wissenschaftliche Buchgesellschaft

Flitner, Andreas/Giel, Klaus (Hrsg.) (1964): Wilhelm von Humboldt. Werke in fünf Bänden. Bd. IV. Darmstadt: Wissenschaftliche Buchgesellschaft

Frühwald, Wolfgang (1991): Humanistische und naturwissenschaftlich-technische Bildung: die Erfahrung des 19. Jahrhunderts. In: Frühwald et al. (1991): 73-111

Frühwald, Wolfgang et al. (Hrsg.) (1991): Geisteswissenschaften heute. Frankfurt am Main: Suhrkamp

Fuchs, Walther Peter/Schieder, Theodor (Hrsg.) (1975): Aus Werk und Nachlaß. Bd. 4: Vorlesungseinheiten. München: Oldenbourg

Fuhrmann, Manfred (1997): Wilhelm von Humboldt und die Berliner Universität. In: Bachmaier et al. (Hrsg) (1997): 29-45

Füssel, Marian (2010): Akademische Aufklärung. Die Universitäten des 18. Jahrhunderts im Spannungsfeld von funktionaler Differenzierung, Ökonomie und Habitus. In: Hardtwig (2010): 47-73

Geldsetzer, Lutz (1974): Die Geisteswissenschaften - Begriff und Entwicklung. In: Rombach (1974): $141-150$

Grimm, Jacob/Grimm, Wilhelm (1854-1971): Deutsches Wörterbuch. 33 Bde. Leipzig: S. Hirzel Verlag

Hamann, Julian (2009): Der Preis des Erfolges. Die ,Krise der Geisteswissenschaften` in feldtheoretischer Perspektive. Bamberg: University of Bamberg Press

Hamann, Julian (2011): Phasen und Formen der Öffnung. Texte und Kontexte des geisteswissenschaftlichen Bildungsdiskurses. In: Angermüller et al. (2011). Im Erscheinen

Hardtwig, Wolfgang (1985): Krise der Universität, studentische Reformbewegung (1750 bis 1819) und die Sozialisation der jugendlichen deutschen Bildungsschicht. In: Geschichte und Gesellschaft 11.2.155-176

Hardtwig, Wolfgang (1990): Geschichtskultur und Wissenschaft. München: DTV

Hardtwig, Wolfgang (Hrsg.) (2010): Die Aufklärung und ihre Weltwirkung. Göttingen: Vandenhoeck \& Ruprecht

Hegel, Georg Wilhelm Friedrich (1907[1807]): Phänomenologie des Geistes. Leipzig: Verlag der Dürr'schen Buchhandlung

Hegel, Georg Wilhelm Friedrich (1955[1822/1837]): Vorlesungen über die Philosophie der Weltgeschichte, Bd. 1: Die Vernunft in der Geschichte. Hamburg: Felix Meiner 
Hegel, Georg Wilhelm Friedrich (1990[1816]): Über den Vortrag der Philosophie an Universitäten. In: Müller (1990): 284-290

Helferich, Christoph (1989): Hegel, Georg Wilhelm Friedrich. In: Lutz (1989): 316-326

Hirschberger, Johannes (1976): Geschichte der Philosophie, Bd. 2: Neuzeit und Gegenwart. Freiburg/ Basel/Wien: Herder

Hockerts, Hans Günter (Hrsg.) (2003): Neue Deutsche Biographie, Bd. XXI. Berlin: Duncker \& Humblot

Hockerts, Hans Günter (Hrsg.) (2005): Neue Deutsche Biographie, Bd. XXII. Berlin: Duncker \& Humblot Humboldt, Wilhelm von (1956[1793]): Über das Studium des Altertums und des griechischen insbesondere. In: Flitner (1956): 12-27

Humboldt, Wilhelm von (1956[1810]): Über die innere und äußere Organisation der höheren wissenschaftlichen Anstalten in Berlin. In: Anrich (1956): 375-386

Humboldt, Wilhelm von (1960[1793]): Theorie der Bildung des Menschen. In: Flitner et al. (1960): $234-240$

Humboldt, Wilhelm von (1960[1821]): Ueber die Aufgabe des Geschichtsschreibers. In: Flitner et al. (1960): 585-606

Humboldt, Wilhelm von (1964a[1809]): Antrag auf Errichtung der Universität Berlin, Juli 1809. In: Flitner et al. (1964): 113-120

Humboldt, Wilhelm von (1964b[1809]): Bericht der Sektion des Kultus und Unterrichts an den König, Dezember 1809. In: Flitner et al. (1964): 210-238

Humboldt, Wilhelm von (2008): Schriften zur Sprache. Neu-Isenburg: Wunderkammer Verlag

Humboldt, Wilhelm von (2008[1814]): Über die Bedingungen, unter denen Wissenschaft und Kunst in einem Volke gedeihen. In: Humboldt (2008): 916-921

Jahn, Bruno (2008): Wolf, Friedrich August. In: Vierhaus (2008): 721-722

Jarausch, Konrad H. (1981): Die neuhumanistische Universität und die bürgerliche Gesellschaft 18001870. Eine quantitative Untersuchung zur Sozialstruktur der Studentenschaften deutscher Universitäten. In: Probst (1981): 11-57

Jarausch, Konrad H. (Hrsg.) (1983): The transformation of higher learning 1860-1930. Expansion, Diversification, Social Opening and Professionalization in England, Germany, Russia and the United States. Stuttgart: Ernst Klett

Jarausch, Konrad H. (2004): Der Lebensweg der Studierenden. In: Rüegg (2004b): 301-322

Jeismann, Karl-Ernst (Hrsg.) (1989): Bildung, Staat, Gesellschaft im 19. Jahrhundert. Stuttgart: Franz Steiner Verlag

Jeismann, Karl-Ernst/Lundgreen, Peter (Hrsg.) (1987): Handbuch der deutschen Bildungsgeschichte. Bd. III: 1800-1870. Von der Neuordnung Deutschlands bis zur Gründung des Deutschen Reiches. München: C. H. Beck

Kant, Immanuel (2005[1798]): Der Streit der Fakultäten. Hamburg: Felix Meiner Verlag

Kauppi, Niilo (1996): French Intellectual Nobility. Institutional and Symbolic Transformations in the Post-Sartrian Era. Albany: State University of New York Press

Keisinger, Florian/Seischab, Steffen (2003): Wozu Geisteswissenschaften? Kontroverse Argumente für eine überfällige Debatte. Frankfurt am Main/New York: Campus

Keller, Reiner (2005): Wissenssoziologische Diskursanalyse als interpretative Analytik. In: Keller et al. (2005): 49-75

Keller, Reiner (2008): Wissenssoziologische Diskursanalyse. Grundlegung eines Forschungsprogramms. Wiesbaden: VS Verlag für Sozialwissenschaften 
Keller, Reiner/Hirseland, Andreas/Schneider, Werner/Viehöver, Willy (Hrsg.) (2005): Die diskursive Konstruktion von Wirklichkeit. Zum Verhältnis von Wissenssoziologie und Diskursforschung. Konstanz: UVK

Keller, Reiner/Truschkat, Inga (2011): Anwendungen der Wissenssoziologischen Diskursanalyse. Wiesbaden: VS Verlag für Sozialwissenschaften. Im Erscheinen

Klinge, Matti (2004): Die Universitätslehrer. In: Rüegg (2004b): 113-143

Koselleck, Reinhart (1991): Wie sozial ist der Geist der Geisteswissenschaften? In: Frühwald et al. (1991): 112-141

Krieg, Matthias (Hrsg.) (1997): Universitas in theologia - theologia in universitate. Festschrift für Hans Heinrich Schmid zum 60. Geburtstag. Zürich: Theologischer Verlag

Lachmann, Karl (1826): Der Nibelunge Not mit der Klage. Berlin: G. Reimer

Lachmann, Karl (1827): Die Gedichte Walthers von der Vogelweide. Berlin: G. Reimer

Liessmann, Konrad Paul (2006): Theorie der Unbildung. Die Irrtümer der Wissensgesellschaft. Wien: Paul Zsolnay Verlag

Lutz, Bernd (Hrsg.) (1989): Metzler-Philosophen-Lexikon. Stuttgart: J. B. Metzlersche Verlagsbuchhandlung

Malinowski, Bernadette (Hrsg.) (2006): Im Gespräch: Probleme und Perspektiven der Geisteswissenschaften. München: Vögele

Markner, Reinhard/Veltri, Guiseppe (Hrsg.) (1999): Friedrich August Wolf. Studien, Dokumente, Bibliographie. Stuttgart: Franz Steiner Verlag

Marquard, Odo (1986a): Über die Unvermeidlichkeit der Geisteswissenschaften. In: Marquard (1986b): 98-116

Marquard, Odo (1986b): Apologie des Zufälligen. Philosophische Studien. Stuttgart: Reclam

McClelland, Charles E. (1980): State, Society, and University in Modern Germany 1700-1914. Cambridge: Cambridge University Press

McClelland, Charles E. (1983): Professionalization and Higher Education in Germany. In: Jarausch (1983): 306-320

McClelland, Charles E. (1988): Die deutschen Hochschullehrer als Elite. 1815-1850. In: Schwabe (1988): 27-53

Mittelstraß, Jürgen (1989): Glanz und Elend der Geisteswissenschaften. Oldenburger Universitätsreden. Bd. 27. Oldenburg: Bibliotheks- und Informationssystem der Universität Oldenburg

Muhlack, Ulrich (2003): Ranke, v., Franz Leopold. In: Hockerts (2003): 140-142

Müller, Ernst (Hrsg.) (1990): Gelegentliche Gedanken über Universitäten. Leipzig: Reclam Verlag

Münch, Richard (2009): Globale Eliten, lokale Autoritäten. Bildung und Wissenschaft unter dem Regime von PISA, McKinsey \& Co. Frankfurt am Main: Suhrkamp

Niebuhr, Barthold Georg (1811-1828): Römische Geschichte. 3 Bde. Berlin: Realschulbuchhandlung

Nipperdey, Thomas (1987): Deutsche Geschichte 1800-1866. Bürgerwelt und starker Staat. München: C. H. Beck

Nörr, Dieter (2005): Savigny, v., Friedrich Carl. In: Hockerts (2005): 470-473

Paletschek, Sylvia (2001): Die permanente Erfindung einer Tradition. Die Universität Tübingen im Kaiserreich und in der Weimarer Republik. Stuttgart: Franz Steiner Verlag

Probst, Christian (Hrsg.) (1981): Darstellungen und Quellen zur Geschichte der deutschen Einheitsbewegung im neunzehnten und zwanzigsten Jahrhundert. Bd. 11. Heidelberg: Winter

Ranke, Leopold von (1975[1831/1832]): Idee der Universalhistorie. In: Fuchs et al. (1975): $72-89$

Reinalter, Helmut/Benedikter, Roland (Hrsg.) (1998): Die Geisteswissenschaften im Spannungsfeld zwischen Moderne und Postmoderne. Wien: Passagen-Verlag 
Ringer, Fritz K. (1987): Die Gelehrten. Der Niedergang der deutschen Mandarine 1890-1933. Stuttgart: Klett-Cotta

Ritter, Joachim (1974a): Die Aufgabe der Geisteswissenschaften in der modernen Gesellschaft. In: Ritter (1974b): 105-140

Ritter, Joachim (1974b): Subjektivität. Sechs Aufsätze. Stuttgart: Reclam

Ritter, Joachim (Hrsg.) (1974c): Historisches Wörterbuch der Philosophie. Bd. 3: G-H. Darmstadt: Wissenschaftliche Buchgesellschaft

Rombach, Heinrich (Hrsg.) (1974): Wissenschaftstheorie. Bd. 1: Probleme und Positionen der Wissenschaftstheorie. Freiburg im Breisgau: Herder

Rüegg, Walter (1997): Der Mythos der Humboldtschen Universität. In: Krieg (1997): 155-174

Rüegg, Walter (2004a): Theologie und Geisteswissenschaften. In: Rüegg (2004b): 325-378

Rüegg, Walter (Hrsg.) (2004b): Geschichte der Universität in Europa. Bd. III: Vom 19. Jahrhundert zum Zweiten Weltkrieg (1800-1945). München: C. H. Beck

Savigny, Carl Friedrich (1990[1808]): Rezension von: F. Schleiermacher, Gelegentliche Gedanken über Universitäten im deutschen Sinn. In: Müller (1990): 259-266

Schelsky, Helmut (1971): Einsamkeit und Freiheit. Idee und Gestalt der deutschen Universität und ihrer Reformen. Düsseldorf: Bertelsmann Universitätsverlag

Schilling, Heinz/Wiedemann, Conrad (1989): Wes Geistes Wissenschaften? Zur Stellung der Geisteswissenschaften in Universität und Gesellschaft. Gießen: Ferber'sche Universitätsbuchhandlung

Schleiermacher, Friedrich (1956[1808]): Gelegentliche Gedanken über Universitäten im deutschen Sinn, nebst einem Anhang über neu zu errichtende. In: Anrich (1956): 219-293

Schleiermacher, Friedrich (1977[1838]): Hermeneutik und Kritik. Frankfurt am Main: Suhrkamp

Schmidt-Wellenburg, Christian (2011): Diskursiver Wandel im Fadenkreuz von Wissenssoziologischer Diskursanalyse und Feldanalyse. Der Aufstieg der Managementberatung. In: Keller et al. (2011)

Schmitz, Matthias (1989): Humboldt, Wilhelm von. In: Lutz (1989): 373-377

Schwabe, Klaus (Hrsg.) (1988): Deutsche Hochschullehrer als Elite: 1815-1945. Boppard am Rhein: Harald Boldt Verlag

Schwinges, Rainer Christoph (Hrsg.) (1999): Artisten und Philosophen. Wissenschafts- und Wirkungsgeschichte einer Fakultät vom 13. bis zum 19. Jahrhundert. Basel: Schwabe \& Co. AG

Schwinges, Rainer Christoph (Hrsg.) (2001): Humboldt International. Der Export des deutschen Universitätsmodells im 19. und 20. Jahrhundert. Basel: Schwabe \& Co. AG

Smith, John H. (1988): The Spirit and Its Letter: Traces of Rhetoric in Hegel's Philosophy of Bildung. Ithaca: Cornell University Press

Spranger, Eduard (Hrsg.) (1910): Fichte, Schleiermacher, Steffens über das Wesen der Universität. Leipzig: Verlag der Dürr'schen Buchhandlung

Stark, Werner (1992): Die Formen von Kants akademischer Lehre. In: Deutsche Zeitschrift für Philosophie 40. 5. 543-562

Steffens, Henrik (1910[1809]): Über die Idee der Universitäten. In: Spranger (1910): 205-280

Stichweh, Rudolf (1999): Die soziale Rolle des Professors der philosophischen Fakultät. Ein Fall von Professionalisierung? In: Schwinges (1999): 335-350

Stolberg-Wernigerode, Otto et al. (Hrsg.) (1959): Neue Deutsche Biographie. Bd. IV. Berlin: Duncker \& Humblot

Tenorth, Heinz-Elmar (1987): Lehrerberuf und Lehrerbildung. In: Jeismann et al. (1987): 250-270

Titze, Hartmut (1985): Der Akademikerzyklus. Göttingen: Vandenhoeck \& Ruprecht

Titze, Hartmut (1987): Das Hochschulstudium in Preußen und Deutschland 1820-1944. Datenhandbuch zur deutschen Bildungsgeschichte. Bd. 1,1. Göttingen: Vandenhoeck \& Ruprecht 
Titze, Hartmut (1995): Wachstum und Differenzierung der deutschen Universitäten 1830-1945. Datenhandbuch zur deutschen Bildungsgeschichte. Bd. 1,2. Göttingen: Vandenhoeck \& Ruprecht

Titze, Hartmut (1999): Der Strukturbruch in der höheren Bildung im 19. Jahrhundert. In: Schwinges (1999): 351-374

Vierhaus, Rudolf (2008): Deutsche Biographische Enzyklopädie. München: K. G. Saur

Virchow, Rudolf (1893): Die Gründung der Berliner Universität und der Uebergang aus dem philosophischen in das naturwissenschaftliche Zeitalter. Berlin: Julius Becker

Weber, Wolfgang (1984): Priester der Klio. Historisch-sozialwissenschaftliche Studien zur Herkunft und Karriere deutscher Historiker und zur Geschichte der Geschichtswissenschaft 1800-1970. Frankfurt am Main/Bern/New York: Peter Lang

Wehler, Hans-Ulrich (1998): Zur Situation der Geisteswissenschaften. In: Reinalter et al. (1998): 187-198

Wolf, Friedrich August (1990[1807]): Vorschläge, wie ohne irgendeinen neuen Aufwand statt der jetzt verlorenen zwei am besten dotierten Universitäten eine für hiesige Lande und für ganz Deutschland wichtige Universität von größerer Anlage gestiftet und in kurzer Zeit in Gang gebracht werden könnte. In: Müller (1990): 44-54

Wolf, Friedrich August (1999[1785/1789]): Fragmente zur Einleitung in die Enzyklopädie der Altertumswissenschaft. In: Markner et al. (1999): 48-75 